\title{
The kinetics of metal oxide photoanodes from charge generation to catalysis
}

Sacha Corby, Reshma R. Rao, Ludmilla Steier and James R. Durrant*

Department of Chemistry and Centre for Processable Electronics, Imperial College London, London, W12 OBZ

*Corresponding: j.durrant@imperial.ac.uk

\begin{abstract}
Generating charge carriers with lifetimes long enough to drive catalysis is a critical aspect for both photoelectrochemical and photocatalytic systems and a key determinant of their efficiency. This review addresses the charge carrier dynamics underlying the performance of metal oxides as photoanodes and their ability to drive photoelectrochemical water oxidation, alongside wider comparison with metal oxide function in photocatalytic and electrocatalytic systems. We start by highlighting the disparity between the ps - ns lifetimes of electron and holes photoexcited in bulk metal oxides versus the $\mathrm{ms}-\mathrm{s}$ timescale of water oxidation catalysis. We go on to review recent literature of the dominant kinetic processes determining photoanode performance, namely charge generation, polaron formation and charge trapping, bulk and surface recombination, charge separation and extraction, and finally the kinetics of water oxidation catalysis. With each topic, we review current understanding and note areas of remaining uncertainty or controversy. We discuss the potential for material selection and examine approaches such as doping, nanostructuring, junction formation and/or co-catalyst deposition to enhance performance. Critically, we examine how such performance enhancements can be understood from analyses of carrier dynamics and propose design guidelines for further material or device optimisation.
\end{abstract}




\section{Introduction}

Solar-driven catalysis is a promising route to the synthesis of low-carbon fuels. The most widely studied direct approach to these solar fuels is the photoelectrochemical (PEC) or photocatalytic (PC) splitting of water to generate green hydrogen and, concomitantly, molecular oxygen. However, driving the oxygen evolution reaction (OER) in photo(electro)catalysis is particularly challenging.

Metal oxides have dominated laboratory research on light-driven photocatalysis since the conceptualisation of photoelectrochemical water splitting using $\mathrm{TiO}_{2}$ in the $1970 \mathrm{~s} .{ }^{1,2}$ Their prevailing use predominantly stems from their high stability in strongly oxidising conditions (i.e. during water oxidation), their relative ease of synthesis through various routes (e.g. vapour deposition techniques, solution processing, templated growth), low cost, ${ }^{3-9}$ and high natural abundance (e.g. iron and titanium oxides ${ }^{10}$ ). Additionally, their morphology, thickness and composition can be readily altered for diverse applications and functionalities. ${ }^{11}$

The metal oxides of primary interest for photoelectrochemical water oxidation are semiconductors with optical bandgaps in the visible or near ultraviolet to enable the absorption of solar irradiation. They range from simple binary oxides, such as $\mathrm{TiO}_{2}, \alpha-\mathrm{Fe}_{2} \mathrm{O}_{3}$ and $\mathrm{WO}_{3}$, and ternaries, such as $\mathrm{BiVO}_{4}$ and $\mathrm{SrTiO}_{3}$, to complex, doped quaternary systems and beyond. Photoactive metal oxides are now found in several commercial applications, including photocatalytic environmental remediation ${ }^{6,12}$ and electrochromic windows, ${ }^{13-15}$ yet the application of these materials in PEC or PC devices for water splitting remains primarily lab-based, with pilot scale demonstration for only a few promising devices, such as the recently developed Z-scheme photocatalyst sheets. ${ }^{16,17}$ The absence of commercial photo(electro)catalytic water splitting devices points to the challenges in simultaneously meeting device efficiency and material stability criteria. Compared to traditional semiconductors, many metal oxides are chemically stable, yet their low carrier mobilities, high defect densities, and short charge-carrier lifetimes and diffusion lengths have thus far limited solar-to-fuel efficiencies to below 5\%, even in the most promising oxide-based PEC and PC systems. Herein, we elucidate the key kinetic challenge for water oxidation catalysis on metal oxides: how to extend the lifetime of the inherently short-lived charges generated by photoexcitation of metal oxides to drive the much slower kinetics of interfacial water oxidation. Addressing this kinetic challenge with minimal energetic loss is central to increasing the efficiency of solar-to-fuel devices.

\section{The kinetic challenge}

Driving redox reactions with metal oxide semiconductors can be considered as a series of steps on progressively longer timescales (Fig. 1). Light absorption generates an excited state, promoting electrons from the filled valence band (VB) to the empty conduction band (CB) and enabling charge transport through the otherwise relatively insulating material. Owing to the strong ionic character of metal oxides, the photoexcited charges tend to interact strongly with the lattice to form polaron states, also sometimes referred to as self-trapping. Localisation on lattice sites can encourage further trapping at defect states. Although trapped charges can be extracted under some conditions, ${ }^{18-21}$ there are multiple recombination pathways that result in the loss of these charges (decay to the ground state) over broad timescales between charge generation and catalysis, including both bulk and surface recombination processes. ${ }^{22}$ The kinetic competition between charge relaxation or recombination and catalysis is arguably the biggest challenge for designing photocatalyst materials and devices, particularly when compared with the design of materials for solar cells where both charges are typically extracted on the nanosecond to microsecond timescale. In PEC systems, at least one charge must drive multi-redox reactions, such as water oxidation, 
occurring on timescales of milliseconds to seconds - approximately six orders of magnitude longer than electron extraction in solar cells. Harnessing photo-generated charges requires efficient spatial separation of electrons and holes such that their annihilation via recombination is minimised. Biological plant photosynthesis employs redox relays to spatially separate the photogenerated charges such that they reach lifetimes sufficient to drive water oxidation. However, this comes at a price: around half the initial energy of the photoexcited states is lost during the charge-separation process. An analogous issue faces metal oxide photoelectrodes, for which the lifetimes of electrons and holes generated in the bulk of the oxide range from a few picoseconds to a few nanoseconds, while the timescales of interfacial water oxidation and reduction are milliseconds to seconds (Fig. 1). This kinetic mismatch is manifested in a typical current-voltage curve as the overpotential required to generate a sufficiently large surface field to drive the spatial separation of charges and reduce recombination losses (Box 1). Therefore, the design of metal oxide photoelectrodes is increasingly using charge-extraction layers and co-catalysts rather than direct semiconductor-liquid junctions to facilitate the spatial separation of charges at a lower energetic cost. ${ }^{23-29}$ We note that meeting this kinetic challenge is a particular challenge for photocatalyst particles, which must operate without an external applied bias to aid charge separation.

\section{BOX 1: Photoelectrochemical water oxidation on metal oxide photoelectrodes}

Semiconductor-liquid junctions used in water oxidation employ n-type photoanodes due to the favourable band bending generated upon electrode immersion. As $n$-type semiconductors typically have higher Fermi energy than the water chemical potential $\left(E_{F}>E_{\text {redox }}\right)$, equilibration is achieved by electron flow away from the semiconductor-electrolyte interface. This loss of majority carriers in the oxide leads to the formation of a space-charge layer $(\mathrm{SCL})$ - a region at the surface of the photoanode that is depleted of electrons, which is balanced by an equal negative charge in the electrolyte at the electrode surface, the Helmholtz layer. The $\mathrm{SCL}$ creates a field that bends the bands and gives the desired directionality of excited charge carrier movement: electrons are repelled by the field and drawn into the bulk whereas holes are driven to the surface (Panel a). Aside from the dielectric constant of the semiconductor (largely invariable) and any external bias applied (variable), the width of the $\mathrm{SCL}$ and strength of the field within is primarily determined by the doping density of the semiconductor; the lower the doping density of the film, the wider the SCL.

Water oxidation in the dark (grey curve, panel b) requires a voltage more positive than $1.23 \mathrm{~V}$ versus the reversible hydrogen electrode $\left(\mathrm{V}_{\mathrm{RHE}}\right)$ (the thermodynamic requirement for water splitting at $25^{\circ} \mathrm{C}$ ). In practice, an additional overpotential, $\eta_{s}$, is required to overcome any kinetic barrier to catalysis at the surface. Additionally, for semiconducting metal oxides, the applied potential must lower the $\mathrm{E}_{\mathrm{F}}$ until it is positive enough to generate sufficient $\mathrm{VB}$ holes to drive water oxidation (grey arrow, panel b). Under illumination, the requirement for an applied positive voltage is reduced by the photo-generation of holes in the VB, i.e., the creation of a photovoltage. Here, a key requirement for the applied voltage is that it is sufficiently positive of the material's flat-band potential to generate an interfacial $\mathrm{SCL}$, resulting in an ideal photoanode performance illustrated by the blue curve for the example of hematite (panel b). In practice, the aforementioned surface overpotential, $\eta_{s}$, is still required to overcome kinetic barriers for catalysis and an additional voltage, $\eta_{\text {rec, }}$ is needed to suppress recombination pathways introduced by the photogeneration of both 
electrons and holes under illumination, thereby necessitating greater overpotentials (orange curve, panel $b$, adapted from Liardet et $a^{30}$ ). Naturally, an earlier onset (smaller $\eta$ ) is desired as this reduces the external energy input required, thereby improving efficiency. The rise in photocurrent in real systems is also not as sharp as the ideal scenario due to these competing recombination processes, which also limit the maximum attainable photocurrent. As such, the J/V curve is a useful metric for initial assessment of any potential metal oxide photoanode but is a result of the multiple underlying competing kinetic processes addressed in this review.
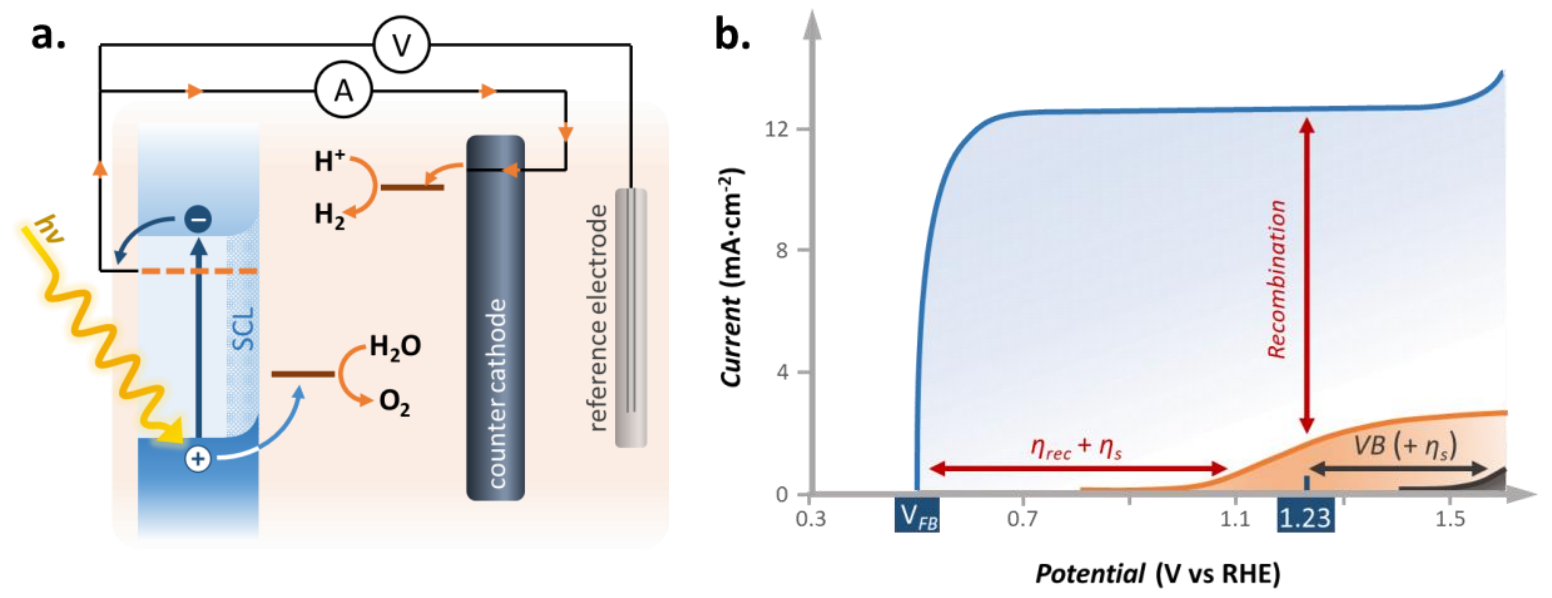

In this review, we address the kinetic challenges facing metal oxide photoanodes from charge generation to catalysis. We begin by discussing the challenges associated with charge carriers in the bulk on femto- to nanosecond timescales and examine the effects of doping, charge trapping and polaron formation. We then focus on kinetics at the semiconductor-liquid junction, examining the role of the surface field and solid-state junctions in aiding spatial charge separation. Finally, we examine the kinetics of water oxidation catalysis, comparing frequently employed kinetic models and drawing parallels with studies of metal oxide electrocatalysts, and touch upon alternative, valueadded oxidation reactions that have the potential to drastically reduce the price of green hydrogen and $\mathrm{CO}_{2}$-neutral fuels. ${ }^{31,32}$ For broader coverage of water splitting oxides, we direct the readers elsewhere. ${ }^{33-37}$ 


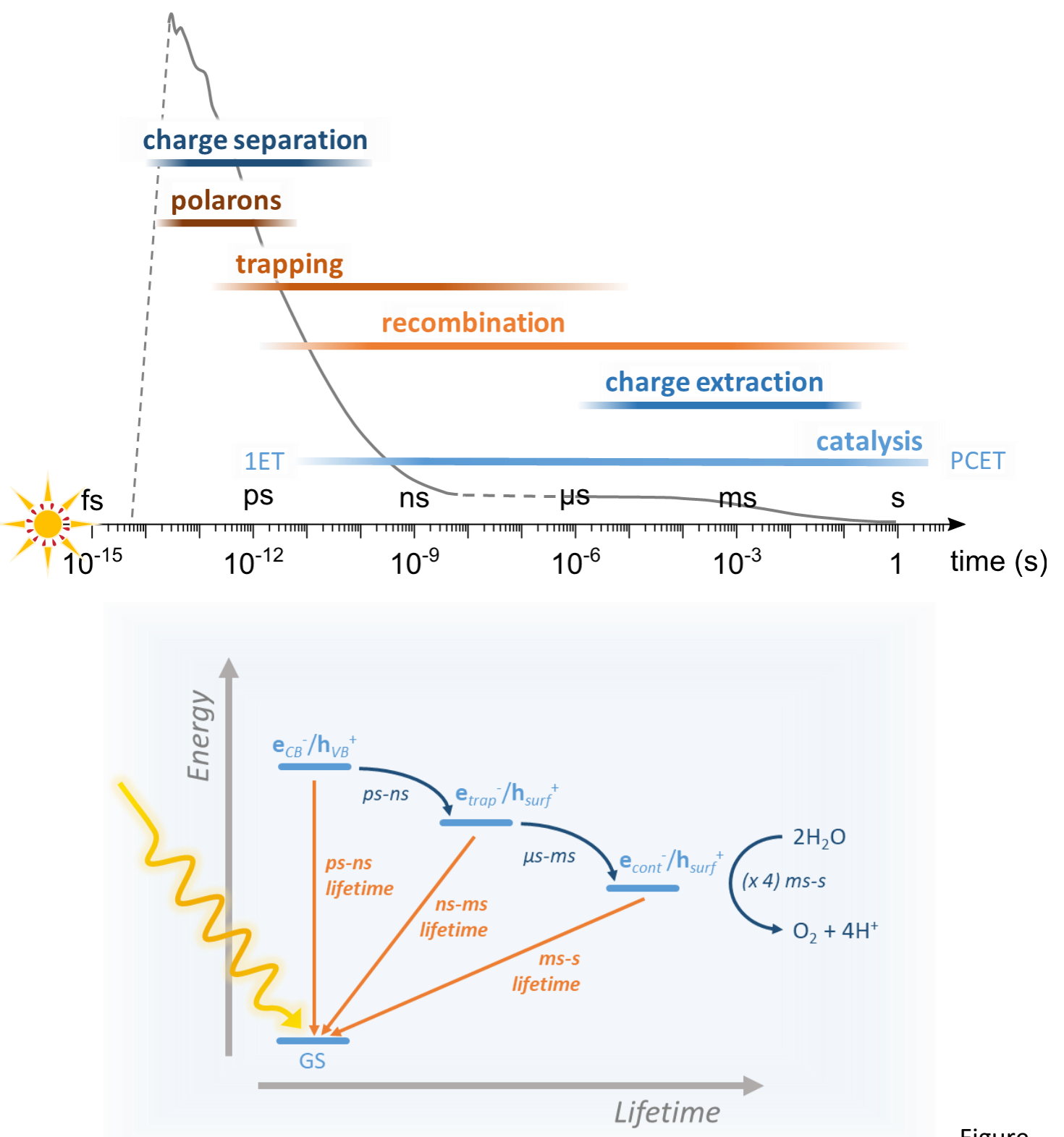

Figure 1:

Timeline of charge carrier dynamics in metal oxide photoanodes from photogeneration to catalysis and the corresponding energy loss associated with lifetime gain. Light absorption generates electron-hole pairs in the metal oxide bulk and space charge layer. These charges must spatially separate and endure for up to milliseconds or even seconds in order to perform useful chemistry (blue processes, upper panel), where 1ET represents reactions with single electron transfers to the electrolyte, as opposed to proton coupled electron transfers (PCET), which are typically much slower. This presents a significant kinetic challenge as recombination occurs over multiple timescales, depleting these charge carriers. Charge localisation in polaron and defect trap states leads to thermally activated charge transport in most oxide materials and reduces the energy of these charges but can also slow recombination (lower panel). An analogous gain in lifetime at a significant energetic cost is central to the success of photosystem II in nature in driving water oxidation. Spectroscopic techniques such as transient absorption can track these charge carrier dynamics, often revealing that only a small fraction of the initial photoexcited carrier population is available for driving catalysis (grey kinetic trace, upper panel). $\mathrm{h}_{\text {surf }}{ }^{+}$is a hole at the surface, $\mathrm{e}_{\text {cont }}{ }^{-}$is an electron at the back contact, $e_{\text {trap }}$ is a trapped electron, and GS is the ground state. 


\section{Charge-carrier generation and separation}

\section{Charge generation, relaxation, and recombination in the bulk}

In the bulk, bandgap photoexcitation of a metal oxide semiconductor is generally assumed to directly generate free electrons and holes owing to the typically high dielectric constants of these materials, although the formation of coulombically bound electron-hole pairs, termed excitons, has been reported. ${ }^{38-42}$ It is striking that the bulk carrier lifetimes of metal oxides vary over several orders of magnitude (Fig. 2), ${ }^{43-47}$ with, for example, $\mathrm{TiO}_{2}$ exhibiting a bulk carrier lifetimes 1,000 -fold longer than those of hematite $\left(\alpha-\mathrm{Fe}_{2} \mathrm{O}_{3}\right)$. It is not well understood why different metal oxides show such divergent bulk-carrier lifetimes. One factor is likely to be doping density; however, systematic studies correlating doping density and bulk carrier lifetimes are strikingly absent from the literature to date for metal oxides (discussed below). Moreover, differences in electronic structure may also be important. ${ }^{48} \mathrm{TiO}_{2}$ is a $d_{0}$ material, with $\mathrm{VB}$ comprising $\mathrm{O}(2 \mathrm{p})$ orbitals and $\mathrm{CB}$ of $\mathrm{Ti}(3 \mathrm{~d})$ orbitals. Several studies have suggested that the incorporation of $d_{0}$ and $d_{10}$ metal centres can produce metal oxides with higher photocatalytic activity than oxides with partially occupied d-orbitals, ${ }^{49-52}$ although the underlying origin of this dependence has not been determined. Regardless, the short bulk carrier lifetimes in hematite are likely one of the key reasons why it has yielded relatively modest solar to hydrogen efficiencies, despite exhibiting favourable band energies for solar light absorption and excellent stability. Notably, short bulk carrier lifetimes are also likely to be a key reason for the relatively poor performance of photovoltaic devices employing visible light absorbing metal oxides ${ }^{53}$.

In most metal oxide semiconductors, photogenerated charges easily interact with the lattice (known as electron-phonon coupling), release heat via relaxation to the band edges (hot carrier cooling) or localise on sub-picosecond timescales..$^{54,55} \mathrm{~A}$ localised charge and its associated lattice deformation are together referred to as a polaron. In many transition metal oxides, charges tend to form 'small' polarons that interact strongly with the lattice. ${ }^{56-59}$ Small polaron formation has been reported in many if not most metal oxides, including in studies of ultrafast electron polaron formation in $\mathrm{TiO}_{2}$, $\mathrm{WO}_{3}, \mathrm{BiVO}_{4}$ and $\alpha-\mathrm{Fe}_{2} \mathrm{O}_{3}{ }^{18,21,57,59,60}$ In hematite, polarons that are smaller than the Fe-Fe interatomic distance form within the first ps after excitation (forming with the same time constant as the typical large drop in TA signal amplitude in Fig. 2b). ${ }^{21}$ Charge trapping in defect sites (Fig. 2c) (which is also associated with charge carrier localisation), is usually associated with an energy loss of a few hundred meV, i.e. a similar magnitude to the energy loss typically associated with polaron formation. ${ }^{21,61}$ Even in the absence of extrinsic chemical dopants, a significant portion of carriers undergo charge trapping into defect sites. In n-type metal oxides commonly used for the OER, oxygen vacancy donor states are the most prevalent chemical defects and their concentrations are often two to three orders of magnitude higher than in classic semiconductors (i.e. $\sim 10^{18}-10^{19} \mathrm{~cm}^{-3}$ in $\mathrm{TiO}_{2}$ and $\mathrm{Fe}_{2} \mathrm{O}_{3}$, and up to $\sim 10^{21} \mathrm{~cm}^{-3}$ for $\left.\mathrm{WO}_{3}\right){ }^{14,20,62}$ Both electron trapping and electron-polaron formation typically result in metal-centred electrons, with the only distinction being whether the metal centre is adjacent to an oxygen vacancy or not. As such, distinguishing these processes is nontrivial (and often not clearly done in literature studies). ${ }^{63}$ It should be noted that metal oxides often exhibit low doping efficiencies, i.e. $\left(\mathrm{N}_{\mathrm{D}}{ }^{+}+\mathrm{e}^{-}\right) / \mathrm{N}_{\mathrm{D}}<1$, indicating that only a limited proportion of dopant sites, $N_{D}$, is ionised in the material bulk (i.e. $N_{D}^{+}$). This low doping efficiency can result in electronic doping densities that are less than the density of chemical doping sites. Low doping efficiencies result from a higher thermal activation required to release a charge carrier from an energetically lower-lying dopant site to the surrounding lattice to enable conductivity. Electron transport in most metal oxides is thermally activated, attributed either to polaron hopping ${ }^{64,65}$ or to thermal excitation from defect trap sites..$^{19,61}$ Therefore, charge-carrier mobilities $(\mu)$ and lifetimes $(\tau)$ in most metal 
oxides are low, resulting in short minority carrier diffusion lengths, $L_{D}=\sqrt{k_{B} T / q \mu \tau}$, where $k_{B}$ is the Boltzmann constant, $T$ is temperature and $q$ is charge. Minority carrier diffusion lengths are typically only 5-100 nm. ${ }^{66}$ It seems that direct bandgap oxide semiconductors suffer much less from small polaron formation and subsequent trapping. For example, $\mathrm{ZnO}$ exhibits band-like electron transport, attributed to the $\mathrm{Zn}$ s-orbital character of its $\mathrm{CB} .{ }^{67}$

Chemical doping with foreign atoms is widely employed in metal oxides to increase the majority (electron) carrier concentration, $n$, to counteract low majority carrier mobilities and increase conductivity $\left(\sigma_{n}=q \mu_{n} n\right.$ ) $)$. A high bulk conductivity is important in, for example, minimising voltage losses associated with bulk electron transport to the back contact of photoanodes. ${ }^{68,69}$ We note, however, that chemical doping can be expected to lower the minority carrier (hole) lifetime, increasing the kinetic challenge to drive catalysis. Both intrinsic defects and extrinsic chemical dopants can introduce electronic states into the bandgap. If energetically close to the CB (or VB), these 'shallow' states can be beneficial, increasing conductivity or enabling the formation of electric fields in the SCL to drive charge separation, and, in some cases, enhance visible light absorption or bulk charge separation. ${ }^{18,20,70,71}$ However, such states also typically result in charge trapping, decreasing the energy (and therefore reactivity) of photogenerated charges and often enhancing charge recombination processes. Charge trapping into intra-bandgap states can mediate charge recombination, also known as Schottky-Read-Hall recombination. Charge trapping can also result in the generation of residual, long-lived, trapped charges, apparent in transient absorption studies as a slow decay phase extending to millisecond timescales. ${ }^{20,72}$

Long-lived trapped charges are typically regarded as too immobile or unreactive to drive useful photoelectrochemistry, as observed, for example, in $\mathrm{N}$-doped $\mathrm{TiO}_{2} \cdot{ }^{73}$ However, in some cases, the lifetime gain associated with shallow trapping has been suggested to aid photoelectrochemical function by stabilising charge separation in e.g. $\mathrm{BiVO}_{4}, \mathrm{WO}_{3}$ and $\mathrm{Fe}_{2} \mathrm{O}_{3} \cdot{ }^{18,61,73,74}$ It was recently reported in $\mathrm{WO}_{3}$ that the ultrafast trapping of photogenerated electrons into ionised oxygen vacancy states can improve the yield of initially separated charges, whilst excessive oxygen vacancy densities accelerate recombination losses, such that optimum photoanode performance is at an intermediate oxygen vacancy density. ${ }^{20}$ It appears that deep, mid-gap states are detrimental to function, whilst shallow trap states can either aid or impede function. ${ }^{17}$ This point was also recently highlighted in analogous studies of charge trapping in carbon nitride photocatalysts with either deep or shallow defect sites. ${ }^{72,75}$ Intertwined with the complex role of dopants and defects such as oxygen vacancies in metal oxides is uncertainty regarding the physical location of these states and, in particular, the potential presence of intra-bandgap surface states at the semiconductor-electrolyte interface, as discussed below.

\section{Defects in the space charge layer (SCL)}

Each intra-bandgap defect can function as either an electron or hole trap depending on its ionisation state. For example, unionised oxygen vacancy states may function as hole traps, but as electron traps when ionised. The Fermi level of the oxide semiconductor determines the fraction of ionised donors, hence, the impact of ionised defects on the J/V curve and on photocatalysis is especially visible in the SCL when varying the working electrode potential (Fig. 3a). It should be noted that, as hole trapping into oxygen vacancy states corresponds to oxidation of neighbouring metal centres (for example, $\mathrm{W}_{\mathrm{ov}}{ }^{5+}$ oxidation to $\mathrm{W}_{\mathrm{ov}}{ }^{6+}$ ), this process is closely analogous to electron-hole recombination and is likely to determine the lifetime of hole minority carriers in n-type metal oxides. Thus, determining the impact of such states upon device performance can be challenging. For 
example, a large concentration of oxygen vacancies in the bulk has been shown to improve the photocurrent density of $\alpha-\mathrm{Fe}_{2} \mathrm{O}_{3}$ photoanodes attributed to a higher electron conductivity $(\sigma \propto$ $\left.\mathrm{N}_{\mathrm{D}}^{+}\right) .{ }^{76}$ However, in the same study, a large oxygen vacancy concentration in the SCL was shown to increase the overpotential for the OER photocurrent onset. On the other hand, recent hematite research has shown that increasing oxygen vacancy densities at bulk crystallographic interfaces can significantly boost bulk conductivity and improve hole collection, dramatically enhancing performance. ${ }^{77}$ Similarly, other defects such as $\mathrm{Ti}$ dopant states cause confusion. $\mathrm{Ti}$ is widely considered to improve conductivity in $\alpha-\mathrm{Fe}_{2} \mathrm{O}_{3}$ photoanodes, ${ }^{78,79}$ but there are also suggestions that the electronic states formed may act as shallow traps that mediate recombination. ${ }^{80}$ Different degrees of effect have also been observed upon elemental doping of $\mathrm{BiVO}_{4}$, with even larger variations in the assigned rationale. ${ }^{34,68,81}$ Hence, defects can be a curse or a blessing, depending on their concentration, position (bulk, SCL, surface) and oxidation state. This is especially visible in our recent study on $\mathrm{La}, \mathrm{Rh}: \mathrm{SrTiO}_{3}$ photocatalyst sheets, where the oxidation state of the Rh dopant shows a potential-dependence in the SCL without the La co-dopant. When in its $4+$ state, Rh acts as an electron trap, whereas when it is fixed to its $3+$ state through a more negative applied potential or the La co-dopant, this trap ceases to exist because of a reorganisation of the electronic structure. $^{82}$

\section{Charge transport to the semiconductor-liquid junction}

The movement of electrons and holes through the photoanode is largely governed by two processes: drift and diffusion. Drift is the directional charge movement under an applied field and thus is the dominant motion of charges within the SCL. For an n-doped photoanode (Fig. 3a), the electric field within this depleted $\mathrm{SCL}$ region drives electrons towards the photoanode bulk and holes towards the surface to react with the electrolyte. This directional movement drives a spatial charge separation, resulting in an increase in carrier lifetime. Diffusion of charge carriers is driven by concentration gradients and may also contribute to charge transport, particularly in the absence of strong fields. Diffusion-driven transport is therefore more significant in the bulk of the photoanode, beyond the SCL. However, as elucidated above, the thermally activated transport in most oxide semiconductors leads to very short diffusion lengths, such that largely only the SCL contributes to the majority of the OER photocurrent generated from metal oxide photoanodes. ${ }^{18,83}$ Short diffusion lengths typically require the use of nanostructured materials to minimise the distance photogenerated carriers have to diffuse to reach the SCL while still maximising light absorption. As discussed above, carrier lifetimes vary substantially between metal oxides. Similarly, experimental measurements of charge carrier mobilities show wide divergences, ${ }^{65}$ in some cases for the same material. For example, Mott Schottky calculations of doping concentrations from electrochemical impedance spectroscopy (EIS) on $\mathrm{BiVO}_{4}$ disagree with concentrations calculated from Hall-effect measurements on the same crystal, possibly owing to the indirect nature of EIS calculations, difficulties accurately determining the ultra-low conductivities in metal oxides with Hall-effect instruments, or complications regarding sub-unity doping efficiencies. ${ }^{84}$ With regards to this last point, most dopant sites can be ionised in the SCL during EIS measurements, resulting in charge carrier densities that are likely to exceed those utilising the Hall-effect to access the material bulk. ${ }^{84}$ Differences in calculated doping concentrations can also be corroborated with disagreements in measured charge carrier diffusion lengths, mobilities, and lifetimes. In $\mathrm{BiVO}_{4}$, for example, comparisons of front and back incident-photon-tocurrent efficiency measurements resulted in reported majority carrier diffusion lengths of $10 \mathrm{~nm},{ }^{85}$ whereas time-resolved microwave conductivity measurements determined the diffusion length of both carriers to be $\sim 70 \mathrm{~nm}$, with lifetimes $\sim 40 \mathrm{~ns} .{ }^{86}$ These divergent results for the same metal oxide demonstrate the likely limitations in comparing properties of different oxides. Furthermore, none of 
these results agree with recently reported lifetimes and mobilities of electrons and holes observed in transient absorption spectroscopy measurements of $\mathrm{BiVO}_{4}$, which show rapid hole trapping/recombination within 20 ps. ${ }^{61,87-89}$ Aside from variations in technique sensitivity, one reason for the different values calculated may be the nature of the species tracked in each measurement, that is, whether lattice relaxation is accounted for, whether exclusively free charges or trapped charges are monitored, and whether the technique better probes carriers generated in the bulk or in the SCL. Furthermore, for some metal oxides, charge-carrier mobility has been reported to be anisotropic due to anisotropic lattice spacing. ${ }^{84,90}$ Anisotropic electron and hole mobilities have been reported to, for example, aid spatial charge separation and enhance the performance of $\mathrm{BiVO}_{4}$ photocatalysts. ${ }^{91} \mathrm{~A}$ unifying model to account for these variations would likely lead to convergence of the reported carrier lifetimes. In any case, it is clear that bulk recombination losses severely affect the attainable photocurrent in many state-of-the-art metal oxides.

To increase the surface-to-bulk ratio, various morphologies and synthetic methods have been introduced, such that most state-of-the-art photoanodes exhibit some degree of nanostructuring. ${ }^{92-}$ ${ }^{95}$ The extent to which nanostructuring is required depends primarily on the carrier diffusion lengths. For example, hematite presents many of the desired qualities for a water oxidation photoanode, with a predicted maximum solar-to-hydrogen efficiency of $15.4 \%{ }^{96}$; however, the maximum attained solar-to-hydrogen efficiency is typically much less, $\sim 1-2 \%,{ }^{97}$ as the majority of photogenerated charges are lost to recombination. ${ }^{80,83}$ This is largely due to the very short bulk recombination timescales (on the order of 5-10 ps) of hematite photoanodes, ${ }^{98,99}$ resulting in hole diffusion lengths of only 2-4 nm. ${ }^{44,66,83}$ We note here that even in a $10 \mathrm{~nm}$ thick, depleted hematite film, only $30-40 \%$ of the maximum theoretical photocurrent was attained, likely stemming from strong electronphonon interactions accelerating non-radiative recombination losses. ${ }^{21,83}$ Alternatively, one can move to materials with longer bulk carrier lifetimes and minority carrier diffusion lengths. For example, the 100-1,000-fold increase in bulk carrier lifetimes for $\mathrm{BiVO}_{4}$ and $\mathrm{TiO}_{2}$ relative to $\alpha-\mathrm{Fe}_{2} \mathrm{O}_{3}$ (Fig. 2b) is likely to be the reason that nanostructuring is critical for efficient $\mathrm{Fe}_{2} \mathrm{O}_{3}$ photoanodes but less so for $\mathrm{BiVO}_{4}$ and $\mathrm{TiO}_{2}$. Indeed, near unity quantum efficiencies have been reported for water splitting by micrometre-sized aluminium-doped $\mathrm{SrTiO}_{3}$ photocatalyst particles, ${ }^{100-102}$ indicating that bulk recombination losses in this material are strongly suppressed. Although the reasons for this recombination suppression are unresolved, they are likely related to the near-intrinsic nature (central mid-gap Fermi level) of this material. 
a.

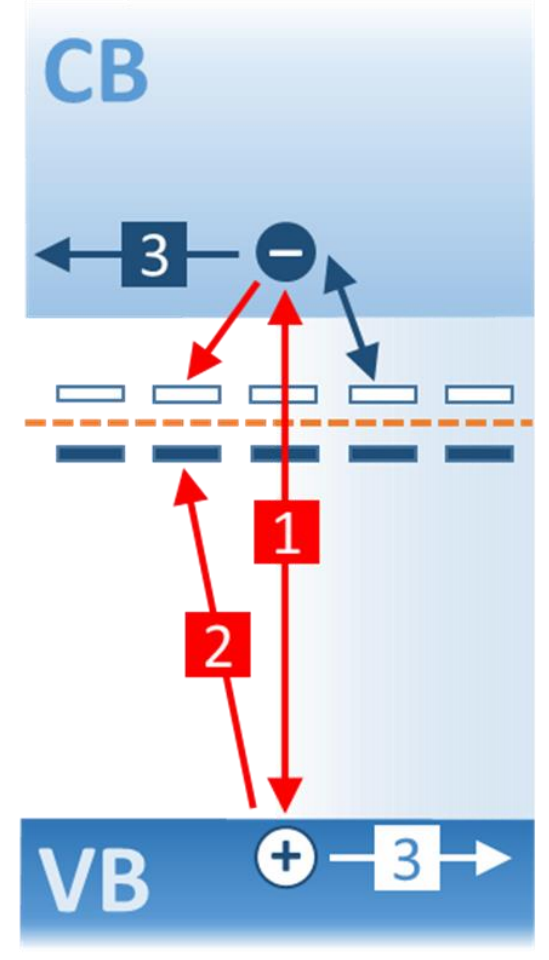

b.

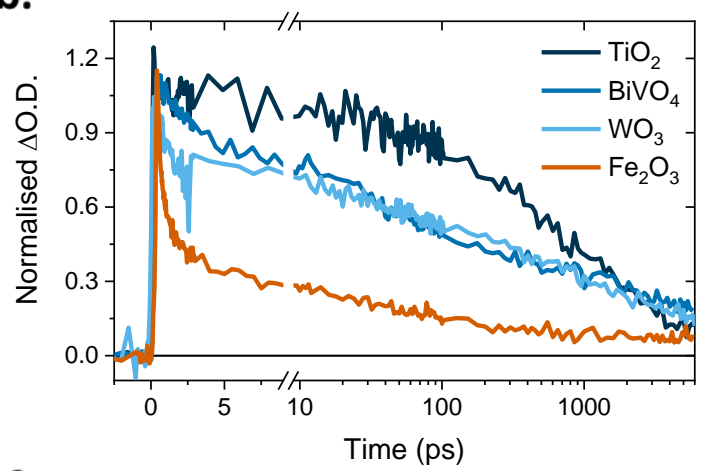

c.

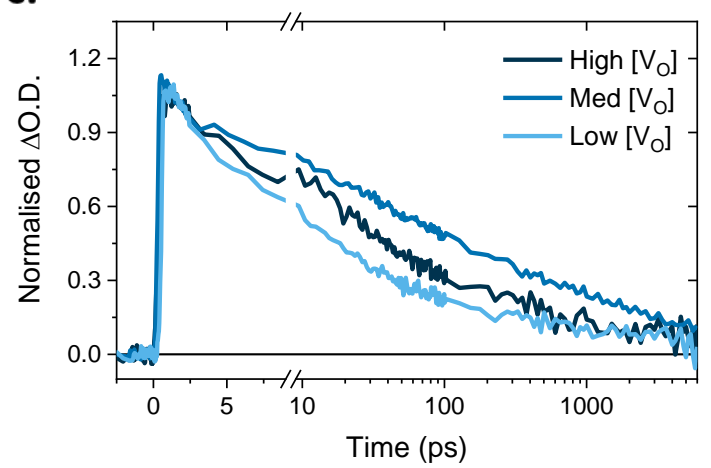

Figure 2: Carrier movement in the bulk (a) A simplified band diagram indicating the main recombination processes (red arrows) that occur in the semiconductor bulk after photoexcitation of an electron (-) from the valence (VB) to conduction band (CB). Route 1: band-to-band recombination (geminate or non-geminate). Route 2: trapping and subsequent recombination via defect/doping states. White boxes are ionised defect states which may trap electrons, and blue states are unionised (filled) states which may trap holes. Route 3: the desired movement of charge carriers, either directly via $C B / V B$ states or via defect/doping states. (b) Transient absorption spectroscopy data under $\mathrm{N}_{2}$, probed at $1100 \mathrm{~nm}$, exemplifying the difference in bulk carrier lifetimes in four different metal oxides. The change on optical density ( $\Delta$ O.D.) is normalised at 1 ps. (c) Transient absorption data on picosecond to nanosecond timescales for $\mathrm{WO}_{3}$ samples with different concentrations of oxygen vacancies $\left(\left[\mathrm{V}_{\mathrm{O}}\right]\right)$, demonstrating how such defects can alter carrier lifetimes. ${ }^{20}$ 
a.

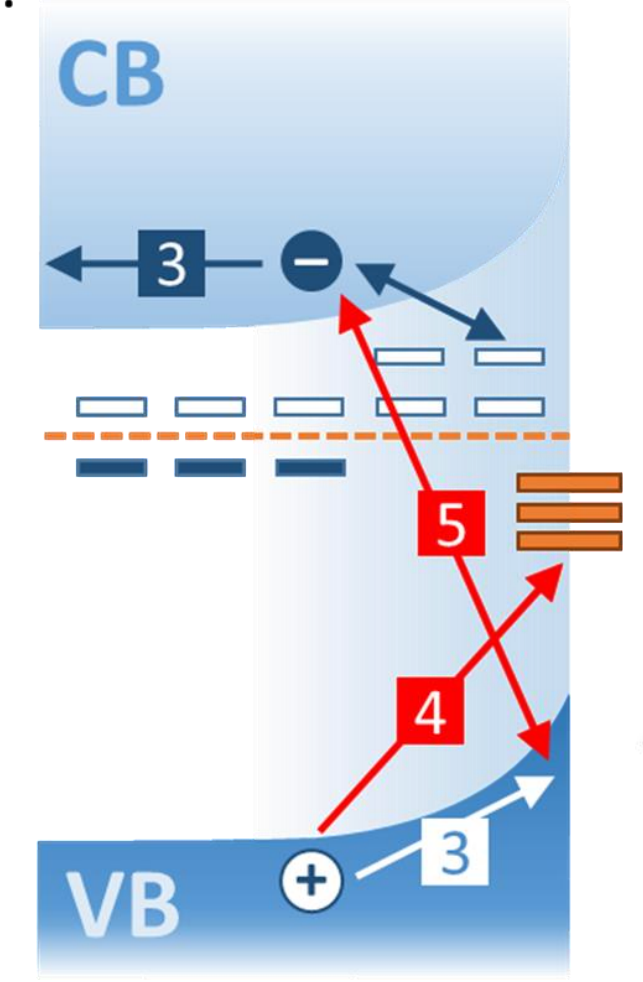

b.
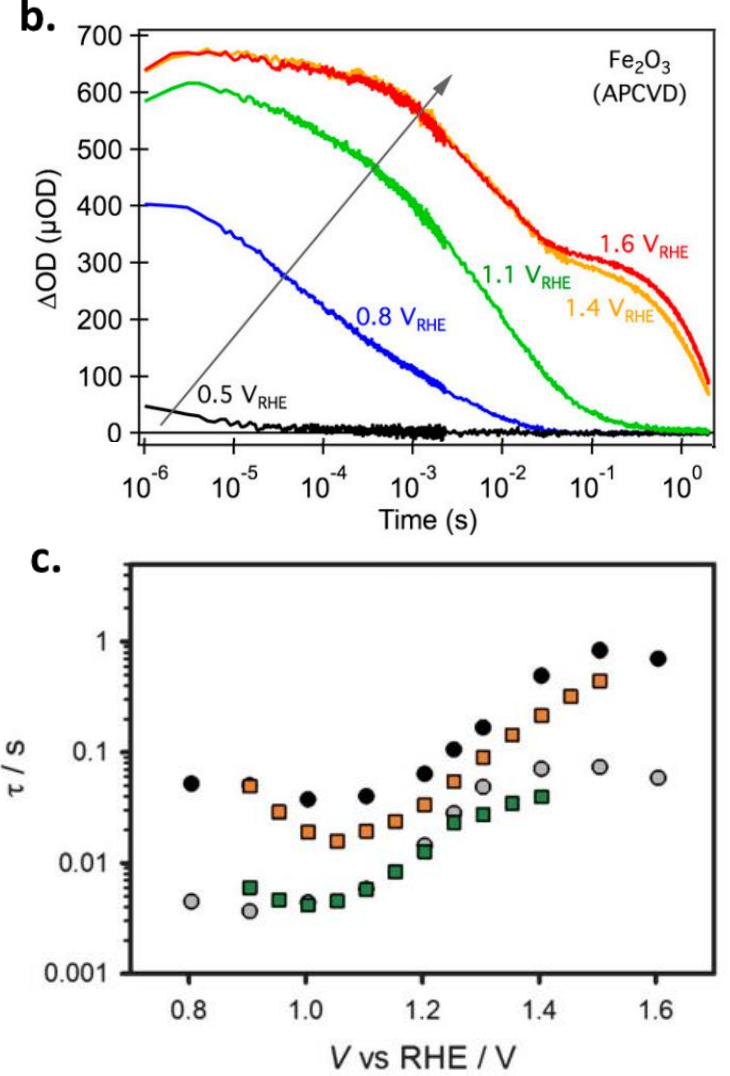

Figure 3: Carrier movement at the surface. (a) A simplified band diagram indicating the main charge separation and recombination processes (red arrows) that occur in the space-charge layer after photoexcitation. Route 4: hole trapping to surface states formed by termination of the oxide lattice at the semiconductor-electrolyte interface. Route 5: back electron-hole recombination between electrons in the conduction band and holes accumulating at the interface to react with water. Route 3 is the desired movement of charge, as illustrated in Figure 2. (b) Transient absorption decays for hematite holes under water oxidation conditions and different applied potentials. Higher applied bias results in a greater number of longer-lived holes. The onset of water oxidation for this electrode was observed at $\sim 1 \mathrm{~V}_{\mathrm{RHE}} .{ }^{103}$ (c) Fitted lifetimes for cathodic current decays in hematite generated when light is turned off. Two different lifetimes have been extracted from a double exponential fit of the cathodic decay, where the black and grey circles represent the slow and fast processes, respectively. The calculated lifetimes for surface state charging-discharging at 1 sun (green squares) and at 0.1 sun (orange squares), taken from impedance spectroscopy are also shown. ${ }^{104}$ 


\section{Gaining lifetime to drive catalysis}

\section{Gaining lifetime at the semiconductor-liquid junction}

Bulk carrier lifetimes in metal oxides are typically in the range of a few picoseconds to a few nanoseconds, while the timescale of water oxidation on metal oxide surfaces is in the range of milliseconds to seconds. Therefore, the lifetime gain required to drive catalysis is typically on the order of $10^{6}-10^{12}$. One means of achieving this lifetime gain is by generating a wide SCL. ${ }^{105,106}$ The electric field present in the SCL drives holes to the surface and electrons to the bulk, thus spatially separating these carriers (Fig. 3a). The maximal lifetime gain from the space-charge fields can be readily estimated from the magnitude of the reduction in electron density at the metal oxide surface relative to the bulk due to the potential drop across the $\mathrm{SCL}\left(\Delta V_{S C L}\right)$, giving a maximum increase in hole lifetime of $\tau_{\mathrm{S}} / \tau_{\mathrm{B}}=e^{\frac{q \Delta V_{S C L}}{k_{B} T}}$. Gaining $10^{9}$ in hole lifetime therefore requires a potential drop across the $\mathrm{SCL}$ of at least $500 \mathrm{mV}$. An alternative, and essentially equivalent, viewpoint is that as the majority (electron) carrier density is strongly suppressed in the SCL relative to the bulk, the lifetime of the minority (hole) charge carriers is substantially increased.

The increase in minority carrier lifetime achieved by generation of an SCL at the metal oxide/electrolyte interface is directly visible in transient absorption measurements as an increase in a long-lived feature when the potential is swept past the OER photocurrent onset potential, as shown here for hematite (Fig. 3b). Analogous increases in hole lifetime have been observed from impedance analyses (Fig. 3c) and these observations are common for many frequently studied metal oxides. ${ }^{22,107-110}$ Typically the increase in carrier lifetime requires a greater positive potential than that predicted from $e^{\frac{q \Delta V_{S C L}}{k_{B} T}}$. For example, this $\Delta V_{S C L}$ argument predicts that a $170 \mathrm{mV}$ increase in applied potential should result in a 1000-fold improvement of minority (hole) carrier lifetime in hematite photoanodes. However, the data in Fig. $3 \mathrm{~b}$ indicates that for the hematite photoanodes studied, such a lifetime improvement requires a $>500 \mathrm{mV}$ increase in applied potential. ${ }^{111}$ We note that direct experimental measurements of the suppression of bulk (ps-ns) bimolecular recombination by applied bias have been very limited to date, ${ }^{18,99}$ most likely due to the high carrier densities generated in such ultrafast spectroscopic experiments screening the SCL fields. ${ }^{18}$ PEC analyses with hole scavengers (which can be rapidly oxidised, and so do not require long surface hole lifetimes) have provided evidence for efficient SCL induced charge separation even at modest applied potentials. ${ }^{112-114}$ Other studies have reported ultrafast hole transfer to the photoanode surface. ${ }^{115-117}$ However, large applied potentials are usually required for surface holes to gain sufficient lifetime to drive the slow OER. In particular, strong SCL fields are required to sufficiently suppress the recombination of accumulated surface holes with bulk electrons, a process referred to as back electron-hole or surface recombination ${ }^{118-120}$ (we note this recombination process should be distinguished from, but is often confused with, recombination via intra-bandgap surface states, which we discuss separately below). As this back electron-hole recombination process results in electrons being drawn into the metal oxide from the back contact, it results in characteristic negative spikes in chopped $\mathrm{J} / \mathrm{V}$ data around photocurrent onset. In $\alpha-\mathrm{Fe}_{2} \mathrm{O}_{3}$ photoanodes, back electron-hole recombination has been observed to compete kinetically with water oxidation catalysis at anodic bias less than $1.3 \mathrm{~V}_{\mathrm{RHE} .}{ }^{121}$ Similar potential requirements have also been reported in $\mathrm{BiVO}_{4}, \mathrm{TiO}_{2}$ and $\mathrm{WO}_{3} \cdot{ }^{19,122-125}$ As such, suppression of back electron/hole recombination is a key consideration for achieving higher photoelectrochemical performance. 
The termination of the periodic lattice at oxide surfaces is expected to result in the formation of chemical defects or surface states that have been widely reported as determining the behaviour of surface charge carriers by acting as traps. ${ }^{126-129}$ In metal chalcogenide nanoparticles, the role of surface states in mediating non-radiative recombination losses has been well established, ${ }^{130-132}$ however, it is not clear whether analogous surface state recombination pathways are prevalent in similarly structured metal oxide systems. Uncertainty over the energy of metal oxide surface states with respect to band-edges and redox potentials has led to some reports of enhanced charge separation ${ }^{133}$ and of water oxidation proceeding through these states, although the latter would only be possible if such states were sufficiently energetic (close to VB) to drive water oxidation. ${ }^{104,134,135} \mathrm{~A}$ larger community report that surface states in metal oxides, as observed for their chalcogenide cousins, enhance recombination losses and are thus detrimental to performance. ${ }^{136-139}$ Indeed, many studies suggest it necessary to passivate any surface states with overlayers to remove the possibility of holes trapping to an energetic level from which they can no longer perform useful chemistry. ${ }^{126-129}$ Impedance analysis has been widely used to identify surface state capacitances, ${ }^{104,140-144}$ although other studies have suggested this capacitance may, in some cases, be mis-assigned. ${ }^{121}$ For example, in a study on hematite conducted by Klahr and Hamann, the authors observed a distinct optical and capacitive feature at $572 \mathrm{~nm}$ which they assigned to a chemically active surface state. ${ }^{145}$ They gave evidence to support this assignment with the addition of an aluminium oxide overlayer that quenches this feature, in line with other reports of $\mathrm{Al}_{2} \mathrm{O}_{3}$ overlayers. ${ }^{129}$ However, subsequent spectroelectrochemical studies have suggested that this absorption feature may be associated with charge trapping in ionised oxygen vacancy states within the $\mathrm{SCL}$, i.e. that this feature does not result from termination of the crystal lattice at the electrolyte interface, but from defect states within the $\mathrm{SCL}^{74}$ An alternative explanation for the passivation effect with alumina may be that $\mathrm{Al}_{2} \mathrm{O}_{3}$ changes the surface band-bending, thereby modulating such trapping processes. It may also be the case that surface state recombination is lessened in metal oxides compared to chalcogenides and other semiconductors due to the almost ubiquitous presence of water to passivate surface defects resulting from surface oxygen vacancies. Indeed transient studies of charge carriers in different $\mathrm{TiO}_{2}$ polymorphs have reported no significant difference in recombination kinetics between mesoporous and dense films, indicating that charge carrier lifetimes in $\mathrm{TiO}_{2}$ are dominated by bulk rather than surface state mediated recombination processes. ${ }^{38,146} \mathrm{~A}$ different interpretation of surface states was presented in a study of $\mathrm{CuWO}_{4}$, in which the authors conclude that there are no permanent or intrinsic surface states but rather that spectroscopic features observed at the surface simply relate to the accumulation of water oxidation intermediates at the photoanode-electrolyte interface. ${ }^{147}$ Indeed, such conclusions agree with the improved performances typical of nanostructured metal oxides compared to their dense counterparts for both PEC and PC systems, suggesting that the benefits of increased surface area (and reduced bulk) greatly outweigh possible losses from surface state recombination.

Other strategies to enhance carrier lifetimes include the manipulation of surface energies or the introduction of ferroelectric fields that may selectively drive charge carriers in a particular crystal direction or towards a particular surface. ${ }^{148-154}$ Alternatively, the doping density of the metal oxide may be further adjusted to improve the built-in field from the SCL. ${ }^{155}$ However, the most widely employed strategy is through the fabrication of solid-state junctions, as detailed below. 


\section{Solid-state junctions and co-catalyst deposition}

Regardless of whether active passivation is required or not, the addition of overlayers and formation of solid-state junctions has shown promising results on charge carrier lifetimes and photoelectrode performances. There are several solid-state junctions used to improve OER photoanode performance (Fig. 4): (i) thin oxide or metal overlayers to extract charge, passivate surface states and/or improve stability; ${ }^{76,129,156,157}$ (ii) oxide/oxide heterojunctions to aid spatial separation of electrons and holes and thereby prolong carrier lifetimes; and (iii) co-catalyst to improve OER. Heterojunctions can be further sub-categorised into type I, II, or III, depending on how the bandedges of the two oxides involved relate to one another. We note that heterojunctions can also be formed at the junction of two phases of the same material, such as rutile and anatase $\mathrm{TiO}_{2},{ }^{158,159}$ where optimised phase junctions can show much higher photocurrent density for water splitting compared to pristine rutile or anatase $\mathrm{TiO}_{2} \cdot{ }^{160}$ An example of a heterojunction that has shown particular promise is the $\mathrm{WO}_{3} / \mathrm{BiVO}_{4}$ photoanode, with multiple publications demonstrating improved photocurrent onsets and attainable maxima with this combination, associated with enhanced charge carrier lifetimes (Fig. 4a,b). ${ }^{88,161-164}$ It is a type II heterojunction, the most commonly used in photoanodes, in which the band-edges are staggered such that the CB and VBedges are cascading from one oxide to the other (Fig. 4a), resulting in band-edge energetic offsets to drive charge separation. The energetics of such heterojunctions may also be modulated by the formation of SCLs at the interface of the two materials resulting from differences in material work functions, as well as by differences in the energetic distributions of trap states in each material. ${ }^{164,165}$ Disagreement over the direction of charge flow at anatase/rutile junctions is a prime example of the complexity of this issue. ${ }^{158,159}$ Overlayers also have the potential to induce band-bending at the junction, which, depending on the relative Fermi levels of the materials involved, may work to further aid or hinder the separation of photogenerated charges (Figure $4 c, d) .{ }^{22}$

Probably the most widely employed solid-state junction strategy to enhance the performance of photoelectrodes and photocatalysts is the deposition of a co-catalyst (Fig. 4e,f). It is, however, not always trivial to determine whether any resulting performance enhancement stems from faster catalysis or from a retardation of competing recombination pathways. Thus, the functionality of an added material as a co-catalyst may be readily misinterpreted, with enhanced performances observed without necessarily enhanced water oxidation kinetics. For example, cobalt phosphate (CoPi) has been widely studied as a co-catalyst for water oxidation on $\mathrm{BiVO}_{4}, \mathrm{WO}_{3}$, and $\alpha-\mathrm{Fe}_{2} \mathrm{O}_{3}$ electrodes, generating notable improvements in performance over the bare electrodes. ${ }^{81,166-168}$ However, several studies have highlighted that the rate constant (or turnover frequency) of water oxidation on CoPi surfaces is actually slower than that on the bare underlying photoanodes. ${ }^{103,122,169}$ The improved performance has therefore, in some cases, been assigned to enhanced charge separation and a resultant reduction in back electron-hole recombination. ${ }^{103,122,169,170}$ A further example of this behaviour is found with $\mathrm{FeOOH}$ catalysed $\mathrm{BiVO}_{4}$, which shows a remarkable enhancement in OER catalysis over bare $\mathrm{BiVO}_{4}$, despite the relatively slow water oxidation kinetics of $\mathrm{FeOOH}$ alone. ${ }^{171}$ Conversely, $\mathrm{IrO}_{2}$, known to be one of the best OER catalysts, was found not to enhance the performance of $\mathrm{BiVO}_{4}{ }^{172}$ These results suggest that the nature of the interface between co-catalyst or overlayer and metal oxide photoanode is critical to performance. Indeed, in a kinetic study on Prussian Blue coated $\mathrm{BiVO}_{4}$, we proposed that the greater covalent nature of bonding at the interface between catalyst and semiconductor allowed for faster charge transfer in comparison to $\mathrm{CoPi}$ coated $\mathrm{BiVO}_{4} \cdot{ }^{173}$ Boettcher et al. have employed dual working electrodes to examine potential variations at the interface between metal oxide semiconductors and oxide-based cocatalysts. ${ }^{168}$ They proposed that the ion-permeability of some catalysts results in the formation of an adaptive Schottky-junction that varies with potential, thus providing another alternative explanation 
as to why some co-catalysts show greater or lesser enhancements on photoanodes than otherwise expected. ${ }^{174}$ We note that co-catalysts are typically able to oxidise water at less oxidising potentials than $\mathrm{n}$-doped semiconducting oxides, and so may be particularly beneficial for enhancing the performance of smaller bandgap photoanodes with valence bands that may otherwise be insufficiently deep to drive water oxidation effectively.

Co-catalyst layers can offer a secondary benefit, independent of whether they function as true rateenhancing catalysts or otherwise. Slow water oxidation kinetics allow the possibility for reactive surface holes to drive alternative reactions to the desired OER. ${ }^{175-177}$ Fortunately, most commonly studied oxides show high Faradaic efficiencies for OER, although not always reaching unity. ${ }^{19}$ The addition of a catalyst can therefore hold the added bonus of improving the Faradaic efficiency, as has been reported for $\mathrm{WO}_{3}{ }^{166,178,179}$ Alternatively, the poor selectivity of some materials for OER can be used to advantage if the water oxidation reaction is replaced by an alternative, desirable oxidative reaction. We revisit this idea in the final section of this review. 
a.

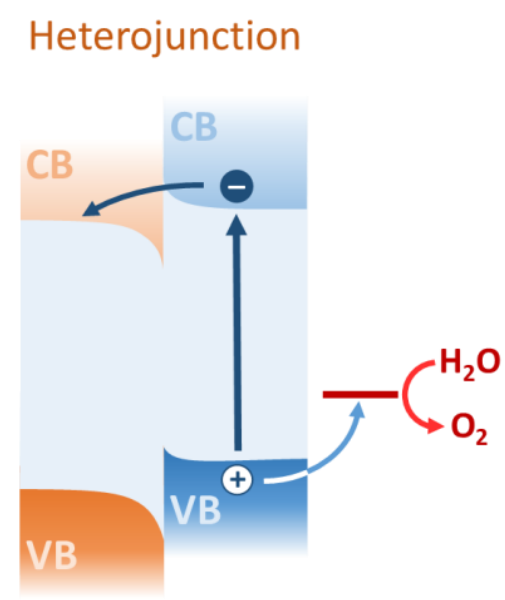

c.

Overlayer

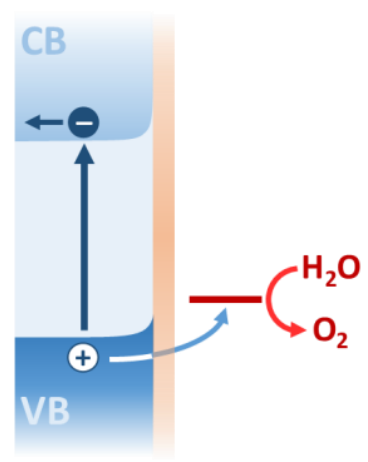

e.

\section{Co-catalyst}

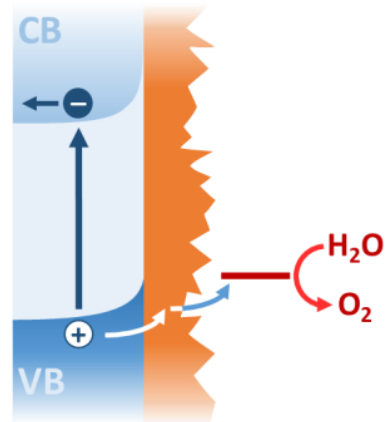

b.

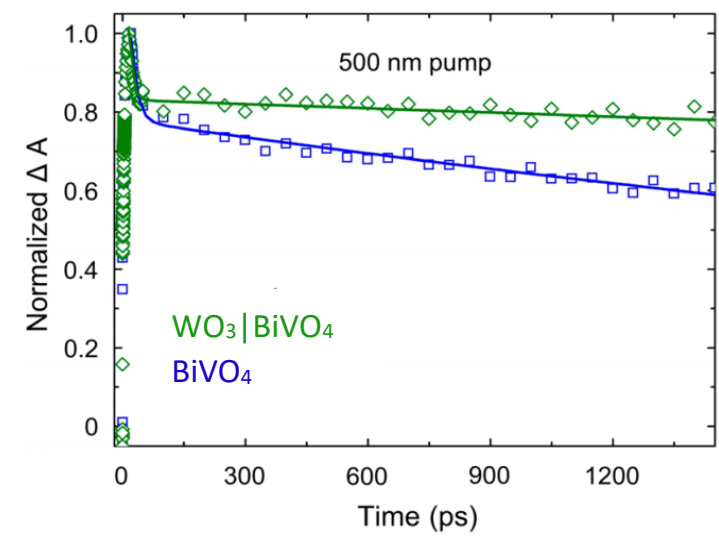

d.

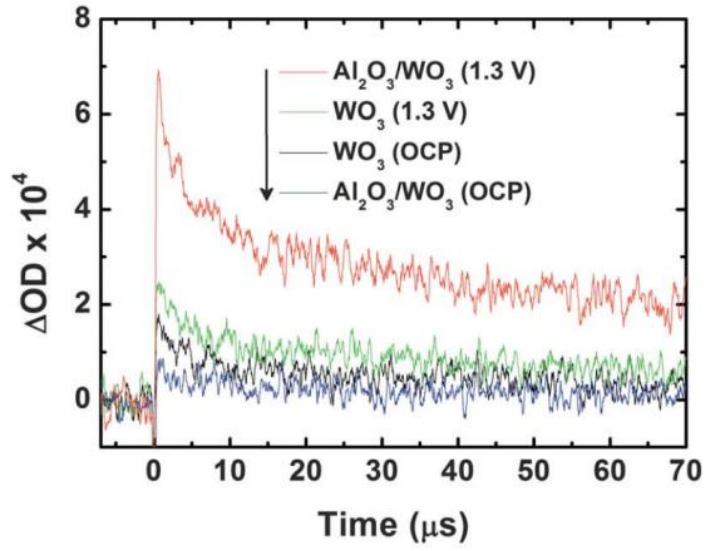

f.

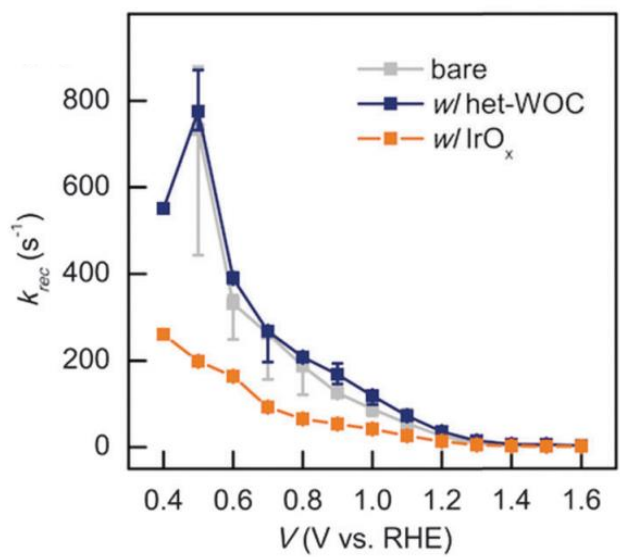

Figure 4: Solid-state junctions. The three main types of semiconductor-solid-state junctions found in metal oxide photoanodes for water oxidation. (a) A type II heterojunction between two metal oxide semiconductors, illustrated with example band-bending for an $n-n$ junction. (b) Ultrafast TA decay data showing the enhanced lifetime attainable when $\mathrm{WO}_{3}$ forms a heterojunction with $\mathrm{BiVO}_{4}$ (green), compared to $\mathrm{BiVO}_{4}$ alone (blue). ${ }^{180}$ (c) A photoanode with an overlayer to passivate the surface. Charges will likely tunnel through this layer. (d) Example of increased yield of long-lived $\mathrm{WO}_{3}$ carriers with $\mathrm{Al}_{2} \mathrm{O}_{3}$ overlayer. ${ }^{181}$ (e) A photoanode with a co-catalyst deposited on top, showing enhanced band bending due to Schottky junction formation. (f) Example of slower surface recombination in hematite photoanodes when an $\mathrm{IrO}_{x}$ catalyst is added. ${ }^{182}$ 


\section{Reaction kinetics of catalysis}

Water oxidation at metal oxide surfaces has been widely reported to occur primarily on ms-s timescales. Although the time scale of water oxidation depends on illumination and applied bias, as discussed in detail below, the reported values typically lie within this range. Tang et al. used transient absorption spectroscopy to show that holes at the surface of $\mathrm{TiO}_{2}$ require between $0.3-1$ second on average to react with water and evolve oxygen. ${ }^{183}$ Subsequent transient absorption studies of other metal oxides have reported similar timescales, ranging from $<1 \mathrm{~ms}$ for $\mathrm{WO}_{3}$ to $\sim 3 \mathrm{~s}$ for $\mathrm{Fe}_{2} \mathrm{O}_{3} .{ }^{19,107,122,184,185}$ Similar timescales for water oxidation have also been reported from operando impedance and photoinduced absorption studies. ${ }^{104,124}$ This ms-s timescale is comparable to the timescales of water oxidation reported for many heterogeneous or molecular water oxidation catalysts. ${ }^{10,186}$ We note that this comparison is perhaps overly flattering to metal oxide photoanode materials, as these drive water oxidation from energetically highly oxidising valence band holes, and therefore operate with much larger effective overpotentials than either photosystem II or most electrocatalysts. In any case, this slow timescale for water oxidation is a major kinetic challenge for efficient photoanode function, requiring the strong suppression of competing surface recombination pathways, as discussed above. It also means that the accumulation of a high density of surface holes is required to generate significant photocurrent densities. For example, for a flat photoanode on which holes can oxidise water in 0.1 second, achieving $10 \mathrm{~mA} \mathrm{~cm}^{-2}$ geometric (equivalent to $\sim 10^{-3} \mathrm{C} \mathrm{cm}^{-}$ 2 geometric) requires a surface hole density of $10^{16} \mathrm{~cm}^{-2}$ geometric (i.e. $100 \mathrm{~nm}^{-2}$ geometric), higher than the density of surface metal sites. One strategy to support such high hole densities per geometric surface area is to use nanostructured electrodes or permeable catalysts, such that the hole density per active area is significantly lower. On the other hand, such high surface hole densities also enable interactions between neighbouring surface holes to drive the multi-redox chemistry required for water oxidation, as we discuss below.

\section{Water oxidation catalysis}

There are multiple approaches to probe water oxidation catalysis on metal oxide surfaces and to determine the best performing photoanodes and catalysts. Many studies examine water oxidation intermediates and their energetics, experimentally ${ }^{187-190}$ or theoretically, ${ }^{191-194}$ to determine the rate limiting catalytic step. ${ }^{195}$ This requires knowledge of which sites or moieties may be catalytically active, and this can be determined using structurally sensitive characterisation techniques in situ. ${ }^{196-}$ ${ }^{200}$ Empirical Brønsted-Evans Polanyi (BEP) relationships are often used to relate kinetics of reactions to simple thermodynamic data; ${ }^{33,201-203}$ however, studies that directly probe water oxidation kinetics are relatively scarce. Here, we highlight advances in direct temporal studies of the kinetics of water oxidation on metal oxide surfaces and their implications for photoanode design and the underlying mechanism of catalysis.

We first assess the relevance of the different parameters influencing water oxidation kinetics. We highlight that the onset of water oxidation for a given oxide under illumination is positive with respect to its flat-band potential by a certain overpotential (n) (Box 1), which may be considered to contain two quantities, $\eta_{\text {rec }}$ and $\eta_{s}$. The first, $\eta_{\text {rec }}$, results from the kinetic challenges discussed above: the need to sufficiently suppress bulk and surface recombination pathways such that surface holes may accumulate with adequate lifetimes to drive water oxidation catalysis. The second, $\eta_{s}$, refers to the potential barrier that holes at the surface may have to overcome to oxidise water. As such, $\eta_{s}$ can be influenced by the nature of the semiconductor (chemical composition of the surface, exposed facets, ${ }^{204-209}$ and even the presence of thermal barriers) ${ }^{184}$ and/or the electrolyte (ions in the supporting electrolyte, ${ }^{176,210,211}$ the $\mathrm{pH}^{212,213}$ etc.). ${ }^{18} \eta_{\mathrm{s}}$ depends on the barrier of hole transfer from 
the valence band to water oxidation intermediates; this is often deemed to be smaller for materials with deeper valence bands, i.e. a larger energetic difference between the redox couple $\left(\mathrm{H}_{2} \mathrm{O} / \mathrm{O}_{2}\right)$ and the valence band. Consequently, a correlation between VB edge and water oxidation rate has been observed for different oxides, which can be likened to lowering the relative reaction barrier by increasing the energy of the 'reactant' relative to the 'transition state'. ${ }^{19,185}$ As shown in Figure $5 a$, the kinetics of water oxidation follows the order $\mathrm{WO}_{3}>\mathrm{TiO}_{2} \sim \mathrm{BiVO}_{4}>\alpha-\mathrm{Fe}_{2} \mathrm{O}_{3}$, which is consistent with the $\mathrm{VB}$ of $\mathrm{WO}_{3}$ being most positive, followed by $\mathrm{TiO}_{2}, \mathrm{BiVO}_{4}$ and $\mathrm{Fe}_{2} \mathrm{O}_{3}$, although there are some studies that argue $\mathrm{TiO}_{2}$ to have the deeper $\mathrm{VB}$ than $\mathrm{WO}_{3} .{ }^{19,185}$ These observations align with work by Butler in which he proposed that $\mathrm{WO}_{3}$ possesses a build-in overpotential for water oxidation. ${ }^{214}$ Despite its suitable VB position, the kinetics of water oxidation for $\mathrm{WO}_{3}$ are still slower $\left(100 \mathrm{~s}^{-1}\right)$ than that achieved in natural photosynthesis by the oxygen-evolving complex (OEC) in Photosystem II $\left(500 \mathrm{~s}^{-1}\right)$, which operates at a relatively small effective overpotential. ${ }^{215,216}$ 
a.

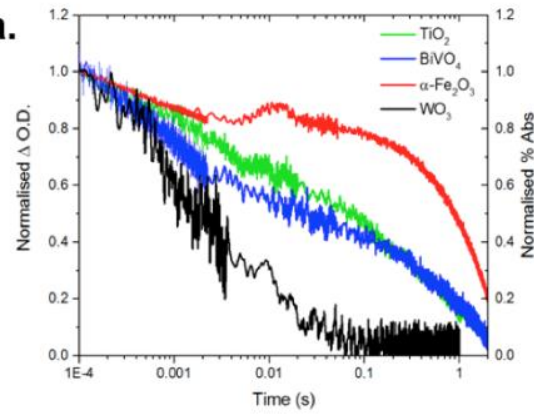

d.

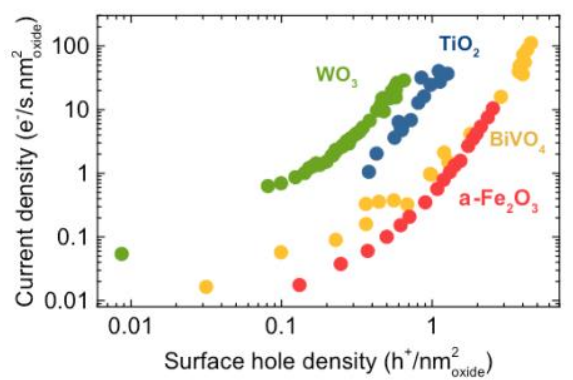

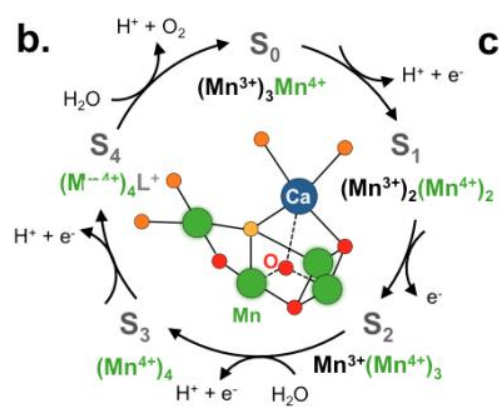
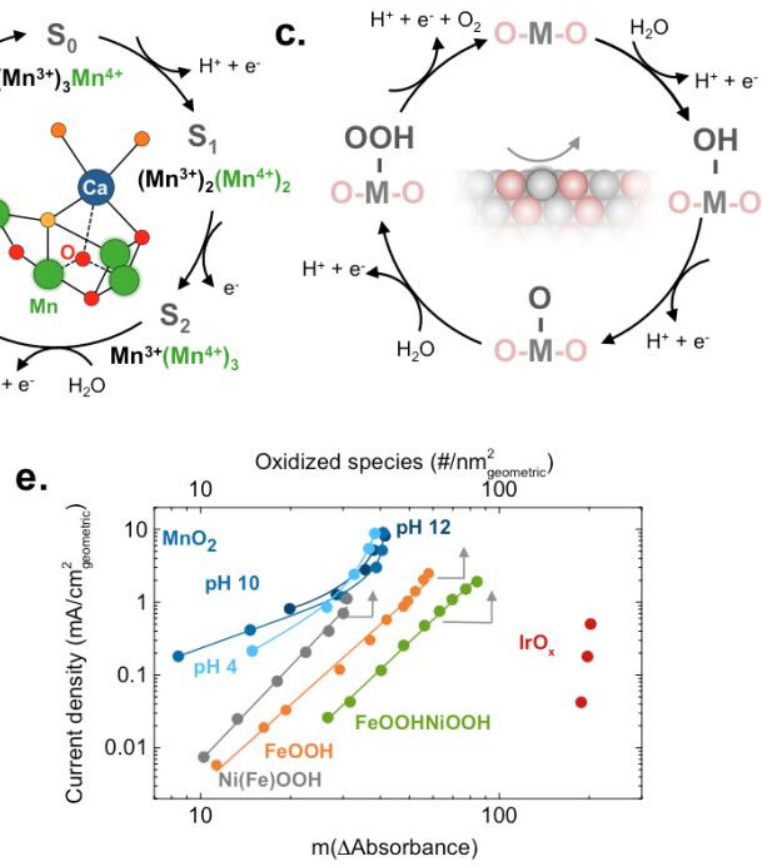

Figure 5: Kinetic models for water oxidation catalysis. (a) Transient diffuse reflectance spectroscopy measurements for holes in $\mathrm{WO}_{3}$ nanoneedles $\left(500 \mathrm{~nm}, \mathrm{pH} \mathrm{1,1.45} \mathrm{V}_{\mathrm{RHE}}\right.$ ) compared to transient absorption decays at photocurrent plateaus for $\mathrm{BiVO}_{4}\left(550 \mathrm{~nm}, \mathrm{pH} 7,1.5 \mathrm{~V}_{\mathrm{RHE}}\right), \mathrm{TiO}_{2}(500 \mathrm{~nm}, \mathrm{pH} 13$, $1.15 \mathrm{~V}_{\mathrm{RHE}}$ and $\alpha-\mathrm{Fe}_{2} \mathrm{O}_{3}\left(650 \mathrm{~nm}, \mathrm{pH} 13,1.5 \mathrm{~V}_{\mathrm{RHE}}\right){ }^{19}$ (b) Multi-site redox levelling mechanism for the water oxidation reaction in OEC of PSII, showing charge accumulation followed by charge rearrangement and evolution of oxygen. (c) Single site mechanism based on the formation of four consecutive electron $/ \mathrm{H}^{+}$transfer steps occurring at the same site. (d) The rate-laws, obtained from SEC-PIAS, for each of the metal oxide photoanodes in (a), taken from ref ${ }^{185}\left(\mathrm{WO}_{3}\right.$, diffuse reflectance system, $\mathrm{pH} 3,1.4 \mathrm{~V}_{\mathrm{RHE}} ; \mathrm{BiVO}_{4}$, transmittance system, $\mathrm{pH} 7$ at $1.7 \mathrm{~V}_{\mathrm{RHE}} ; \alpha-\mathrm{Fe}_{2} \mathrm{O}_{3}$, transmittance system, $\mathrm{pH} 13$ at $1.5 \mathrm{~V}_{\mathrm{RHE}} ; \mathrm{TiO}_{2}$, transmittance system, $\mathrm{pH} 13$ at $1.5 \mathrm{~V}_{\mathrm{RHE}}$ ). The surface holes and currents are normalized to the oxide surface area, accounting for the roughness of the samples. (e) Comparative rate laws for different metal oxide-based electrocatalysts. Lower $\mathrm{x}$-axis is unitless as the extinction coefficient was not known for all samples. $\mathrm{Ni} / \mathrm{FeOOH}$ measurements were made in $\mathrm{pH} 13^{217}, \mathrm{MnO}_{2}$ $(510 \mathrm{~nm}, \mathrm{pH} 4, \mathrm{pH} 10$ and $\mathrm{pH} 12)$ from $\operatorname{ref}^{218}$ and $\operatorname{IrO}_{x}(450 \mathrm{~nm}, \mathrm{pH} 2)$ from ref ${ }^{219}$. The current and oxidized species are normalized to the geometric area of the electrodes.

The Butler-Volmer model (Box 2) has been widely used in electrochemistry to describe interfacial charge transfer across metal/electrolyte interfaces, i.e. where a change in free energy of electrons in the electrode is directly associated with a potential change across the Helmholtz layer, resulting in a reduction in activation energy for the reaction and a potential dependent rate constant. ${ }^{220,221}$ However, for semiconductors that only achieve low carrier densities (relative to metals) at the electrolyte interface, increasing the applied potential primarily increases the accumulation of charge carriers at the interface, and the rate constant may remain relatively invariant. ${ }^{138,222-224}$ In such systems, an alternative population model (Box 2) has been proposed, ${ }^{214,225-227}$ where additional terms to account for the effect of surface ${ }^{228}$ and depletion layer ${ }^{214,225,226,229-231}$ recombination can be included to describe reaction kinetics at the semiconductor/electrolyte interface. In this model, the change in entropy derived from changes in the surface carrier concentration (i.e. a change in chemical potential or free energy, rather than enthalpy) is the dominant factor altering the driving force of the reaction. ${ }^{113,125,232,233}$ 


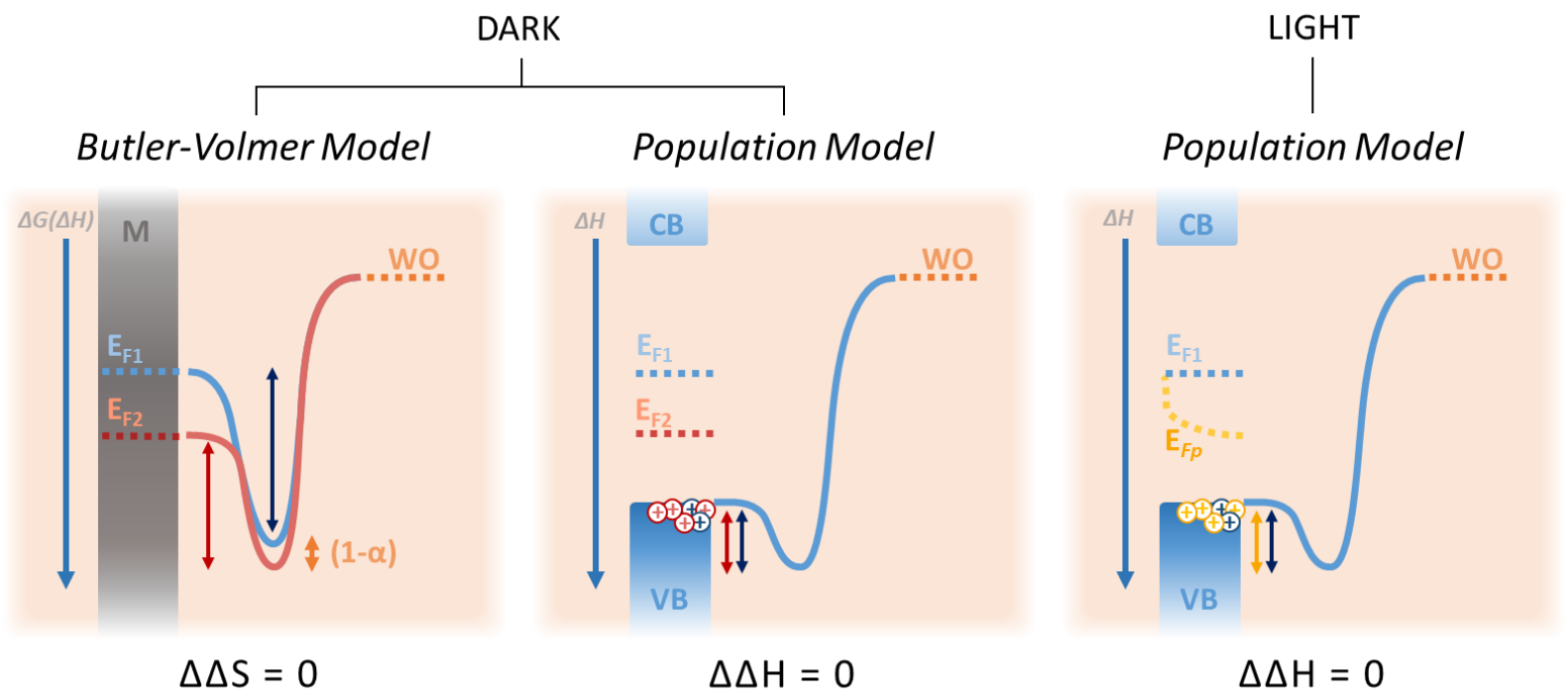

The most frequently employed model to study the current-voltage relationship of an electrode is the Butler-Volmer model, demonstrated for simplicity with a metal-electrolyte interface in left panel of the figure, where a continuum of energy states can be accessed within the metal. ${ }^{220,221}$ In this picture, the application of positive bias lowers the metal electrode Fermi level $\left(\mathrm{E}_{\mathrm{F} 1}\right)$ to a new position $\left(E_{F 2}\right)$ and thus changes the enthalpy of the states performing water oxidation (note free energy change approximately equals enthalpy change in this case). The barrier to water oxidation is thereby lessened (red arrow < blue arrow) and the current increases (the barrier changes with potential but by a smaller factor, 1- $\alpha$, where $\alpha$ is the transfer coefficient). However, while this model is well-suited to metals with a continuum of accessible states, it is arguably less well suited to semiconductors, or indeed to metals with localised rather than band states driving catalysis. Alternatively, a population model can be used (centre panel of the figure) to describe dark catalysis for a semiconductor with its Fermi level in the bandgap. This model is then also applicable under irradiation, substituting the deeper Fermi level for the hole quasi-Fermi level that forms with light (right panel of the figure). Note that, for clarity, we focus here solely on reactive holes at the SCL and do not consider the role of band-bending in this picture, although it is undoubtedly critical in delivering separated charges to the interface. ${ }^{225,234}$ For a photoanode in the absence of Fermi level pinning, increasing the applied potential (centre) OR the light intensity (right) increases the concentration of photogenerated holes at the VB edge. In this population model, the (photo)current generated depends only on the concentration of holes at the surface, with their reactivity increasing with concentration due their resultant loss of entropy rather than an increase in enthalpy. ${ }^{170}$ Thus, in the Butler-Volmer model applied to metals, the rate constant for water oxidation changes with Fermi level, while in the population model applied to semiconductors, the rate constant is invariant and the population (concentration) of charge carriers changes. Similar concepts have been postulated to analytically explain current-voltage relationships, ${ }^{170,214,225,235}$ with additional complexities in the models arising from the treatment of surface reaction rates ${ }^{230,231,236}$ and recombination at the surface ${ }^{228}$ and in the depletion layer. ${ }^{229}$ In this most simple picture, the enthalpy of the states does not change and neither does the kinetic barrier to water oxidation (red arrow = blue arrow) and the current depends only on the population of accumulated species at the interface. This population model is likely to be 
particularly relevant to materials with localised charge carriers, such as the small polarons formed in most metal oxides. 
The population-based model has been used to describe OER kinetics for a range of photoanodes. The simplest version of this model assumes that the water oxidation current density $(J)$ is proportional to hole concentration $\left[\mathrm{h}^{+}\right]$, and first-order rate constants (kwo) are then applied to model behaviour; i.e. $\mathrm{J}=\mathrm{kwo}_{\mathrm{wo}}\left[\mathrm{h}^{+}\right] .{ }^{223,237,238}$ For example, Cummings et al. and others used this assumption to determine the lifetimes of surface trapped holes in hematite photoanodes. ${ }^{109,124,239}$ However, OER is a complex four electron reaction and, depending on the reaction mechanism, the rate or current $(\mathrm{J})$ can, in principle, be proportional to the first, second, third or fourth power of the hole density at the surface $\left(\mathrm{h}^{+}\right)$, i.e. $J=\mathrm{kwo}_{\mathrm{wo}}\left[\mathrm{h}^{+}\right]^{\alpha}$, where $\alpha$ is the order of reaction with respect to surface holes. Furthermore, multiple active sites can be involved. ${ }^{185,240}$ Indeed, rate law analyses on hematite employing operando spectroelectrochemical photoinduced absorption spectroscopy (SECPIAS) have shown a third order dependence of photocurrent on hole generation under one sun irradiation conditions, with a first order water oxidation regime appearing only at low light intensities, Figure $5 \mathrm{~d} .{ }^{233} \mathrm{An}$ order greater than one can be rationalised by either: (1) considering each of the oxidation processes prior to the rate determining step to be in equilibrium with one-another such that they appear equivalent; or (2) a cooperative effect between two or more active sites or species resulting in a multi-site mechanism. For the specific case of hematite exhibiting a reaction order of three, it has been suggested that the rate determining step corresponds to the formation of the $\mathrm{O}-\mathrm{O}$ bond by the chemical oxidation of water driven by three surface holes (i.e. $\mathrm{Fe}(\mathrm{IV})=\mathrm{O}$ species) coming together in a dimeric metal oxide cluster, with the kinetics of lateral surface hole diffusion most likely impacting upon the observed water oxidation kinetics. ${ }^{185,241}$ The activation energy for this rate-determining step was determined to be only $60 \mathrm{meV}$ for the third order reaction, compared to $300 \mathrm{meV}$ for the first order mechanism. ${ }^{185}$ Interestingly, the water oxidation kinetics for equivalent hole densities was found to be independent of the applied electrochemical potential, further supporting the role of hole accumulation in driving water oxidation ${ }^{242,243}$. We note that the surface hole density measured under one sun irradiation in this study was $2 \times 10^{14} \mathrm{~cm}^{-2}$, higher than typically expected surface defect densities, and suggesting that water oxidation may proceed across the whole oxide surface rather than from localised catalytic sites - a conclusion also supported by DFT simulations of reaction mechanism. ${ }^{185}$ Similar third order behaviour has also been reported for $\mathrm{TiO}_{2}$ and $\mathrm{BiVO}_{4}$ photoanodes, alluding to a similar water oxidation mechanism across comparable oxides. ${ }^{185}$ The increase in rate constant with deeper valence band edge (as noted above) can be attributed more energetic surface holes promoting $\mathrm{O}-\mathrm{O}$ bond formation, potentially by increasing the enthalpic driving force for three surface holes to come together to form the triply oxidised species involved in the rate-determining step. Changes to $\mathrm{pH}$ conditions, however, have been shown to alter the mechanism for water oxidation on the same materials, i.e. different rate order, ${ }^{125,244}$ which may be expected when considering the different reaction pathways available in acidic and alkaline media and the predicted changes in surface protonation of the examined oxides. Indeed, faster water oxidation kinetics were observed for hematite photoanodes at $\mathrm{pH}$ greater than its point of zero charge, indicative of proton coupled reaction kinetics. ${ }^{185}$

Rate orders greater than one for water oxidation suggest that metal oxides operate by a similar redox-levelling mechanism proposed for the biological water oxidation enzyme of photosystem II (PSII), via the build-up of multiple oxidising equivalents prior to the $\mathrm{O}-\mathrm{O}$ bond formation, (Fig. 5b) as opposed to a single site mechanism where $-\mathrm{OH},-\mathrm{O}$ and $-\mathrm{OOH}$ intermediates are formed on the same active centre (Fig. $5 \mathrm{c}$ ). ${ }^{216,245}$ In the $\mathrm{Mn}_{4} \mathrm{CaO}_{5}$ cluster of PSII, oxidation and deprotonation steps within a narrow electrochemical potential range (redox levelling) result in the formation of multiple oxidized $\mathrm{Mn}$ states. The eventual formation of oxygen can occur via an oxyl-oxo coupling pathway ${ }^{246,247}$ or by the reaction of a substrate water molecule with a terminal oxyl radical. ${ }^{248-251}$ Such a redox-levelling, multi-site mechanism allows for lower electrochemical overpotentials and relies on $\mu$-oxo bridges that link the metal sites and regulate binding, deprotonation, and oxidation of substrate water molecules. Similar catalytic motifs have been synthesised for electrocatalysts such as $\mathrm{CoOOH},{ }^{252,253} \mathrm{NiOOH},{ }^{254-256} \mathrm{MnOOH}^{257}$ and amorphous ${ }^{258,259}$ and molecular IrO $\times$ clusters, ${ }^{260-262}$ where equivalent oxo-coupling mechanisms have been proposed. We recently employed a similar 
rate law analysis to the one described above, using stepped potential spectroelectrochemistry to study a series of $\mathrm{Ni} / \mathrm{Fe}$ oxyhydroxide $(\mathrm{Ni} / \mathrm{FeOOH})$ electrocatalysts containing different ratios of nickel to iron. ${ }^{217}$ Figure 5 e shows the dependence of the water oxidation current on the density of oxidised states (or the change in absorption) on a log-log plot, where the slope represents the order of the reaction $(\alpha)$ with respect to the oxidised states. Despite observing different oxidised species (Fe- or $\mathrm{Ni}$-based) with different metal ratios, and different dependencies of the population of these species with applied electrochemical potential, we found a reaction order of four on all electrodes (Fig. 5e), suggesting a similarity in the reaction mechanism. Notably, Tafel plots generated from the same data revealed significant differences in the value of the Tafel slope between the catalysts, usually taken as an indicator of different catalytic mechanisms. ${ }^{263}$ However, this discrepancy can be attributed to the significant change in the density of oxidised species observed as a function of potential, which is not accounted for in conventional Tafel analysis. The impact of population densities on performance has similarly been reported by Takashima et al. in their studies of water oxidation on $\delta-\mathrm{MnO}_{2}$ using spectroelectrochemistry in solutions of different $\mathrm{pH}^{218,264}$ They observed that $\delta-\mathrm{MnO}_{2}$ exhibited higher water oxidation activity at higher $\mathrm{pH}$ coincident with an earlier electrochemical potential for the onset for the accumulation of the oxidised species, further indicating the importance of population density in driving water oxidation on electrocatalysts as well as on photoanodes.

The population model is valid only so long as the driving force (photovoltage for photoanodes, voltage for anodes) only changes the entropy (i.e. density) of the oxidised states, with their enthalpy remaining constant. As shown in Fig. 5e, while the kinetics for $\mathrm{MnO}_{2}$ at a low density of oxidised species are well represented by a population model and a reaction order of one, high potential leads to rapidly increasing current densities over a much smaller increase in oxidised species, resembling Tafel behaviour. These results also show that the solution $\mathrm{pH}$ can alter the governing kinetic behaviour at a given density of oxidised species. The kinetics on state-of-the-art $1 \mathrm{IO}_{\mathrm{x}}$ catalysts is more challenging to understand over the entire water oxidation regime, considering the different oxidized species that can be accumulated on the surface and the smaller optical changes detected ${ }^{219,265}$ relative to $\mathrm{Ni}_{x} \mathrm{Fe}_{1-x} \mathrm{OOH}$ and $\mathrm{MnO}_{2}$ (Figure 5e). Interestingly, a recent study on $1 \mathrm{rO}_{x}$ combining pulse voltammetry, X-ray absorption spectroscopy and DFT calculations has proposed that increasing the density of oxidized species lowers the activation energy of the rate determining chemical $\mathrm{O}-\mathrm{O}$ bond formation step, and electrochemical potential only promotes the formation of the oxidized states. Therefore, a deviation from rate-law behaviour can also be possible if the accumulation of oxidized states significantly alters the enthalpy of the states driving water oxidation $^{266,267}$. However, hematite photoanodes, potential independent reaction kinetics, once normalised for surface hole density, have been reported for both methanol and water oxidation, strongly supporting the validity of a population model for this system. ${ }^{242,243,268}$

The applicability of either a Butler Volmer model or a population model depends on the nature of the (photo)electrode, electrolyte, applied potential, and the reaction mechanism; it is probable that in many cases the true kinetic behaviour lies between these two extremes. For example, details of $\mathrm{O}-\mathrm{O}$ bond formation by chemical interaction of two oxo species and the possible deviation in kinetic regime from a population model once all sites are present in their most oxidised form is unknown for both metal oxide photoanodes and electrocatalysts. In addition, band-edge unpinning can occur for photoanodes in the presence of large charge densities, or at high applied potentials, which could allow for the Helmholtz potential drop between the semiconductor and solution to change. In this case, a larger fraction of the potential drop may be experienced across the Helmholtz layer, increasing the likelihood of Butler-Volmer-like behaviour. ${ }^{242,269-271}$ The magnitude of such effects will also depend on the net charge of accumulating species; for example, surface holes on hematite have been suggested to be uncharged $\mathrm{Fe}(\mathrm{IV})=\mathrm{O}$ species, potentially reducing their impact on band-edge unpinning. ${ }^{185,242,272}$ Furthermore, it is important to note that water oxidation is an inner-sphere reaction and the validity of the assumptions for outer-sphere electron transfer models such as the Butler-Volmer theory remains questionable 266,273 . 
Achieving a unified understanding of water oxidation kinetics on (photo)anodes requires advances in both experimental and computational capabilities to account for complex, multi-site interactions and to enable tracking of reaction intermediates at faster timescales, necessary to provide further insight into the reaction mechanisms at play. Temporal resolution of the elementary steps has been carried out on some promising materials using X-ray diffraction and infrared spectroscopy techniques. ${ }^{115,117,274-277}$ For example, in the case of $\mathrm{Co}_{3} \mathrm{O}_{4}$, two distinct intermediates were observed using time-resolved FTIR spectroscopy, a superoxo and an oxo species. ${ }^{275}$ Their temporal behaviour suggested that these two species belong to two different mechanisms, with the formation of the superoxo species corresponding to the fast cycle that involved a cooperative effect between two adjacent $\mathrm{Co}(\mathrm{IV})$ sites, analogous to the $11^{\text {st }} / 3^{\text {rd }}$ order behaviour discussed above for hematite photoanodes. In another study, by combining ultrafast infrared spectroscopy and theoretical calculations for subsurface vibrations on $\mathrm{n}-\mathrm{SrTiO}_{3}, \mathrm{Cuk}$ et al. found that the oxyl intermediate on the $\mathrm{Ti}$ site was generated within picoseconds of charge transfer and was present for at least nanoseconds after generation. ${ }^{117}$ Once decoupled from the lattice, the Ti-O stretch was found to couple to water dynamics, thus highlighting the role of the electrolyte in the eventual formation of the O-O bond. Similarly, advances have been made in theoretical modelling of water oxidation to capture the differences in kinetics that result from a change in available oxidised sites, as well as the energetics of each site, with applied potential. ${ }^{263,278-280}$ By proposing a change in the nature of surface sites as a function of potential under water oxidation conditions, these studies can explain changes in the kinetic regimes observed experimentally, which were previously attributed to different reaction mechanisms using the simplified Tafel analysis. Such studies, combined with direct experimental evidence of the density and nature of surface oxidised states under reaction conditions, may enable a more detailed understanding of water oxidation kinetics. Understanding the underlying kinetics of relevant reaction intermediates for water oxidation, and their dependence on interfacial parameters, brings the community closer to tuning the rate of catalysis to outcompete surface recombination processes, with the potential for dramatically enhancing performance.

\section{Alternative Oxidations}

Water oxidation is, undoubtedly, a kinetically challenging reaction. Improvement in interfacial kinetics requires a detailed understanding of the relevant intermediates, mechanism, and kinetic model that governs the reaction, which can vary largely with the electrode, electrolyte, and regime of operation. This reaction is particularly challenging because four charge transfer processes need to be optimised individually, resulting in inevitable energy losses. This has motivated replacing this halfreaction with other molecules that may be oxidised to give value-added products. Alternative reactions such as methanol, ethanol or glycerol oxidation could be substituted in place of the water oxidation chemistry and provide greater yields at improved turnover frequencies. For example, methanol is widely applied as a hole scavenger, thereby directly demonstrating its relative ease of oxidation compared to water. ${ }^{268,281-284}$ Hematite has been shown to oxidise methanol to formaldehyde (a product of industrial use) with 96\% Faradaic efficiency, while requiring significantly less external bias than the analogous OER from water. ${ }^{268}$ Another common hole scavenger is hydrogen peroxide $\left(\mathrm{H}_{2} \mathrm{O}_{2}\right)$, which is also a possible intermediatery product en route to oxygen evolution from water. ${ }^{285}$ With a greater inherent value than $\mathrm{O}_{2}$ and potential use in medicinal applications, several research groups have examined limiting water oxidation to this, kinetically easier, two electron oxidation product. ${ }^{286}$ Choi and co-workers recently demonstrated the photoelectrochemical conversion of biomass at a $\mathrm{BiVO}_{4}$ photoanode while simultaneously generating hydrogen at the cathode. ${ }^{287} \mathrm{Li}$ et al. have since expanded on this to show how biomass 
conversion on $\mathrm{BiVO}_{4}$ is attainable in non-aqueous media. ${ }^{288}$ Similar biomass conversions have also been successfully implemented in electrochemical hydrogen generation cells, ${ }^{289}$ and Reisner and coworkers have shown efficient conversions of both biomass and plastic waste to hydrogen. ${ }^{290,291}$ Alternatively, Du et al. have proposed chloride oxidation as a replacement reaction, which, rather than generating a significantly higher value product, could provide benefits by allowing sea water to be used in place of valuable purified water in (photo)electrochemical cells. ${ }^{292}$ Zhang et al. on the other hand have employed hematite photoanodes for arene $\mathrm{C}-\mathrm{H}$ amination. ${ }^{293}$ From a kinetic perspective, many of these alternative oxidations are typically faster than water oxidation, thereby reducing the lifetime challenge for efficient function, and thus reducing the applied voltage required to drive these reactions and the concommitant evolution of molecular hydrogen. 


\section{Future Perspective}

Although metal oxides are the most widely studied material class for photo(electro)catalytic solar fuel devices, their solar energy conversion efficiencies ( $3 \%$ for $\mathrm{PEC}^{26}$ and $1 \%$ for $\mathrm{PC}^{16}$ ) remain significantly below those required for commercial application. Their performance limitations are primarily due to the kinetic mismatch between bulk charge carrier lifetime and the timescale of interfacial catalysis, resulting in recombination of most photoexcited electrons and holes that could have driven formation of green $\mathrm{H}_{2}$ from water splitting. We summarise below some material design guidelines identified in this review to address this kinetic challenge and highlight areas of remaining uncertainty or controversy. We focus here only on guidelines based on kinetic considerations - there are already excellent reviews covering, for example, the need to select materials with appropriate band energies and stabilities. ${ }^{5,7,8,33,92,194,206,294-296}$

Guideline 1: Reduce the magnitude of the kinetic challenge by selecting or synthesising a light absorbing metal oxide with a longer bulk charge carrier lifetime. A long carrier lifetime reduces bulk recombination losses and increases the minority carrier diffusion length, reducing the need for nanostructuring. For example, $\mathrm{BiVO}_{4}$ exhibits a $\sim 100$-fold longer bulk carrier lifetime than $\mathrm{Fe}_{2} \mathrm{O}_{3}$, which is a key reason behind its generally superior performance. However, understanding the factors that determine bulk carrier lifetimes in metal oxides remains an important challenge, alongside improved rationale for doping (see below), and exploration into the role of $d_{0} / d_{10}$ materials, which appear to favour longer intrinsic lifetimes.

Guideline 2: Reduce the magnitude of the kinetic challenge by selecting a metal oxide or co-catalyst with faster water oxidation kinetics. Faster water oxidation kinetics reduce the density of positive charge carriers (holes) that must accumulate at the photoanode surface to drive the required flux of water oxidation, thereby suppressing surface (back electron-hole) recombination losses. Faster water oxidation kinetics tend to correlate with deeper metal oxide VB edges, although a larger bandgap reduces light harvesting efficiency. Some, but not all, co-catalysts (molecular or heterogeneous) exhibit faster water oxidation kinetics (i.e. high turnover frequencies) at lower potentials. Co-catalyst addition may enable some otherwise inactive, visible light absorbing metal oxides to drive water oxidation. The oxidation of alternative substrates to water (e.g. alcohols, chloride etc.) may also reduce this kinetic challenge.

Guideline 3: Employ material energy offsets to aid the spatial separation of charge carriers. The band bending induced by an applied bias is the simplest way to drive charge separation but requires an energy input that reduces efficiency. Junction formation, such as metal oxide/co-catalyst or type II semiconductor heterojunctions can aid the spatial separation of charges, reducing or removing the need for an applied bias. Internal material fields, including those generated by ferroelectric fields or differences in surface energies on different crystal facets, can also aid charge separation, as can energetic offsets induced by local variations in material composition or crystallinity.

Guideline 4: Tune dopant densities for optimum performance. Photoanode performance requires ntype doping to enhance bulk conductivity and enable SCL formation. However, a high doping density can be expected to reduce the minority carrier (hole) lifetime, and so accelerate bulk recombination losses (although there is little direct evidence of this to date), as well as reduce the width of the SCL. Tuning of dopant energetics could modulate the doping efficiency and so modulate the impact of the dopant on minority carrier lifetime or SCL formation. Spatial modulation of doping density (e.g. $\mathrm{n}$ type in the bulk, $p$-type at the surface) may also enhance performance. 
Guideline 5: Tune, and most likely minimise, polaron relaxation and charge trapping. Most metal oxides exhibit strong polaron localisation/relaxation and are highly defective with significant charge trapping into localised defect sites. Both processes reduce charge carrier mobilities and are energetic loss processes; charge trapping can also mediate charge recombination losses. However shallow charge trapping can also retard charge recombination and aid photocatalytic function (as long as the trapped carriers retain sufficient energy and mobility to drive the desired reactions). Many defects may also dope the material, such as oxygen vacancy doping in many metal oxide photoanodes.

While substantial progress has been made in understanding how charge carrier dynamics modulate PEC and PC performance, and how these dynamics can be enhanced by appropriate materials design, many aspects of these kinetic studies remain unresolved. Progress towards addressing areas of poorer understanding is being accelerated by the development of new experimental techniques, such as time resolved X-ray spectroscopy and in situ/operando spectroscopic studies. ${ }^{297}$ For example, such techniques could probe the movement of holes at the semiconductor surface, delivering insights that could resolve the ongoing controversy regarding the susceptibility of n-type metal oxides to surface-state mediated recombination processes. Likewise, the role of water or oxygen in surface passivation and the relationship between bulk and surface defects remain areas of high interest that would benefit from further study. Perhaps the largest area of uncertainty lies in the kinetics of multi-redox catalysis, as we discussed above and in Box 2. The kinetics of catalysis are not only scientifically interesting, but also technologically important, with the likely non-linear dependence of these kinetics upon charge accumulation and/or interfacial energetics impacting directly upon the shape of the photoanode J/V curve. Similarly, the non-linear dependence of both catalysis and bimolecular recombination kinetics upon light intensity is likely to impact strongly upon PEC and PC performance as a function of solar intensity, particularly for solar concentrator applications. We emphasise that the kinetic processes discussed herein as underlying PEC and PC water splitting have close parallels with those in other applications, including PEC and PC $\mathrm{CO}_{2}$ reduction, photocatalytic pollutant degradation, and water electrolysis. Furthermore, the kinetic challenges discussed herein for metal oxides are also critical for most other material classes currently under investigation for PEC and PC applications, including metal nitrides, carbon nitrides, conjugated polymers, and molecular photosystems. As such, we hope that this review provides useful insights and inspiration to those interested in harnessing sunlight to drive chemical reactions and reduce our impact upon our environment.

\section{Acknowledgements}

We would like to acknowledge financial support from the European Research Council (project Intersolar 291482) and the European Union's Horizon 2020 research and innovation program under grant agreement 732840-A-LEAF. L.S. acknowledges her Marie Skłodowska Curie fellowship funding (H2020-MSCA-IF-2016, Grant No. 749231). We further acknowledge the UKRI Strategic Priorities Fund for funding. Additional thanks to Camilo A. Mesa, Michael Sachs, Benjamin Moss and Shababa Selim for their thoughts and suggestions.

\section{References}

1 Fujishima, A. \& Honda, K. Electrochemical Photolysis of Water at a Semiconductor Electrode. Nature 238, 37-38, doi:DOI 10.1038/238037a0 (1972). 

Society 115, 199, doi:10.1149/1.2411080 (1968).

3 Ahmed, B., Kumar, S., Ojha, A. K., Donfack, P. \& Materny, A. Facile and controlled synthesis of aligned WO3 nanorods and nanosheets as an efficient photocatalyst material.

Spectrochimica Acta Part A: Molecular and Biomolecular Spectroscopy 175, 250-261, doi:https://doi.org/10.1016/i.saa.2016.11.044 (2017).

Huang, Z.-F., Pan, L., Zou, J.-J., Zhang, X. \& Wang, L. Nanostructured bismuth vanadate-based materials for solar-energy-driven water oxidation: a review on recent progress. Nanoscale 6 , 14044-14063, doi:10.1039/C4NR05245E (2014).

Alexander, B. D., Kulesza, P. J., Rutkowska, I., Solarska, R. \& Augustynski, J. Metal oxide photoanodes for solar hydrogen production. Journal of Materials Chemistry 18, 2298-2303, doi:10.1039/B718644D (2008).

Huang, Z. F. et al. Tungsten Oxides for Photocatalysis, Electrochemistry, and Phototherapy. Advanced Materials 27, 5309-5327, doi:doi:10.1002/adma.201501217 (2015).

Sivula, K. \& van de Krol, R. Semiconducting materials for photoelectrochemical energy conversion. Nature Reviews Materials 1, 15010, doi:10.1038/natrevmats.2015.10 (2016). Yang, Y. et al. Progress in Developing Metal Oxide Nanomaterials for Photoelectrochemical Water Splitting. Advanced Energy Materials 7, 1700555, doi:10.1002/aenm.201700555 (2017).

9 Wang, Q. et al. Scalable water splitting on particulate photocatalyst sheets with a solar-tohydrogen energy conversion efficiency exceeding 1\%. Nature Materials 15, 611-615, doi:10.1038/nmat4589 (2016).

Roger, I., Shipman, M. A. \& Symes, M. D. Earth-abundant catalysts for electrochemical and photoelectrochemical water splitting. Nature Reviews Chemistry 1, 1-14, doi:10.1038/s41570-016-0003 (2017).

11 Yu, X., Marks, T. J. \& Facchetti, A. Metal oxides for optoelectronic applications. Nature Materials 15, 383-396, doi:10.1038/nmat4599 (2016).

12 Bignozzi, C. A. et al. Nanostructured photoelectrodes based on WO3: applications to photooxidation of aqueous electrolytes. Chem Soc Rev 42, 2228-2246, doi:10.1039/c2cs35373c (2013).

13 Niklasson, G. A. \& Granqvist, C. G. Electrochromics for smart windows: thin films of tungsten oxide and nickel oxide, and devices based on these. Journal of Materials Chemistry 17, 127156, doi:10.1039/B612174H (2007).

14 Deb, S. K. Opportunities and challenges in science and technology of WO3 for electrochromic and related applications. Solar Energy Materials and Solar Cells 92, 245-258, doi:10.1016/j.solmat.2007.01.026 (2008).

15 Granqvist, C.-G. Electrochromic materials: out of a niche. Nature materials 5, 89-90 (2006).

16 Wang, Q. et al. Scalable water splitting on particulate photocatalyst sheets with a solar-tohydrogen energy conversion efficiency exceeding 1\%. Nature materials 15, 611-615 (2016).

17 Wang, Q. \& Domen, K. Particulate Photocatalysts for Light-Driven Water Splitting: Mechanisms, Challenges, and Design Strategies. Chemical Reviews 120, 919-985, doi:10.1021/acs.chemrev.9b00201 (2020).

18 Corby, S. et al. Charge Separation, Band-Bending, and Recombination in WO3 Photoanodes. The Journal of Physical Chemistry Letters, 5395-5401, doi:10.1021/acs.jpclett.9b01935 (2019).

19 Corby, S. et al. Water Oxidation and Electron Extraction Kinetics in Nanostructured Tungsten Trioxide Photoanodes. Journal of the American Chemical Society 140, 16168-16177, doi:10.1021/jacs.8b08852 (2018).

20 Corby, S., Francàs, L., Kafizas, A. \& Durrant, J. R. Determining the role of oxygen vacancies in the photoelectrocatalytic performance of WO3 for water oxidation. Chemical Science 11, 2907-2914, doi:10.1039/C9SC06325K (2020). 
21 Pastor, E. et al. In situ observation of picosecond polaron self-localisation in $\alpha$-Fe2O3 photoelectrochemical cells. Nature Communications 10, 3962, doi:10.1038/s41467-01911767-9 (2019).

22 Cowan, A. J. \& Durrant, J. R. Long-lived charge separated states in nanostructured semiconductor photoelectrodes for the production of solar fuels. Chemical Society Reviews 42, 2281-2293, doi:10.1039/C2CS35305A (2013).

23 Verlage, E. et al. A monolithically integrated, intrinsically safe, 10\% efficient, solar-driven water-splitting system based on active, stable earth-abundant electrocatalysts in conjunction with tandem III-V light absorbers protected by amorphous TiO 2 films. Energy \& Environmental Science 8, 3166-3172 (2015).

$24 \mathrm{Hu}$, S. et al. Amorphous TiO2 coatings stabilize $\mathrm{Si}, \mathrm{GaAs}$, and $\mathrm{GaP}$ photoanodes for efficient water oxidation. Science 344, 1005-1009 (2014).

25 Paracchino, A., Laporte, V., Sivula, K., Grätzel, M. \& Thimsen, E. Highly active oxide photocathode for photoelectrochemical water reduction. Nature materials 10, 456-461 (2011).

26 Pan, L. et al. Boosting the performance of $\mathrm{Cu} 2 \mathrm{O}$ photocathodes for unassisted solar water splitting devices. Nature Catalysis 1, 412-420 (2018).

27 Sivula, K., Formal, F. L. \& Grätzel, M. WO3-Fe2O3 Photoanodes for Water Splitting: A Host Scaffold, Guest Absorber Approach. Chemistry of Materials 21, 2862-2867, doi:10.1021/cm900565a (2009).

28 Maeda, K. \& Domen, K. Solid solution of $\mathrm{GaN}$ and $\mathrm{ZnO}$ as a stable photocatalyst for overall water splitting under visible light. Chemistry of Materials 22, 612-623 (2010).

29 Tilley, S. D., Cornuz, M., Sivula, K. \& Grätzel, M. Light-Induced Water Splitting with Hematite: Improved Nanostructure and Iridium Oxide Catalysis. Angewandte Chemie 122, 6549-6552, doi:10.1002/ange.201003110 (2010).

30 Liardet, L., Katz, J. E., Luo, J., Grätzel, M. \& Hu, X. An ultrathin cobalt-iron oxide catalyst for water oxidation on nanostructured hematite photoanodes. Journal of Materials Chemistry $A$ 7, 6012-6020 (2019).

$31 \mathrm{Na}$, J. et al. General technoeconomic analysis for electrochemical coproduction coupling carbon dioxide reduction with organic oxidation. Nature communications 10, 1-13 (2019).

32 Vass, Á., Endrődi, B. \& Janáky, C. Coupling electrochemical carbon dioxide conversion with value-added anode processes: an emerging paradigm. Current Opinion in Electrochemistry (2020).

33 Hunter, B. M., Gray, H. B. \& Müller, A. M. Earth-Abundant Heterogeneous Water Oxidation Catalysts. Chemical Reviews 116, 14120-14136, doi:10.1021/acs.chemrev.6b00398 (2016).

34 Park, Y., McDonald, K. J. \& Choi, K.-S. Progress in bismuth vanadate photoanodes for use in solar water oxidation. Chemical Society Reviews 42, 2321-2337, doi:10.1039/C2CS35260E (2013).

35 Seo, J., Nishiyama, H., Yamada, T. \& Domen, K. Visible-Light-Responsive Photoanodes for Highly Active, Stable Water Oxidation. Angewandte Chemie International Edition 57, 83968415, doi:10.1002/anie.201710873 (2018).

36 Singh, A. \& Spiccia, L. Water oxidation catalysts based on abundant 1st row transition metals. Coordination Chemistry Reviews 257, 2607-2622, doi:https://doi.org/10.1016/i.ccr.2013.02.027 (2013).

37 Dau, H. et al. The Mechanism of Water Oxidation: From Electrolysis via Homogeneous to Biological Catalysis. ChemCatChem 2, 724-761, doi:10.1002/cctc.201000126 (2010).

38 Sachs, M., Pastor, E., Kafizas, A. \& Durrant, J. R. Evaluation of Surface State Mediated Charge Recombination in Anatase and Rutile TiO2. The Journal of Physical Chemistry Letters 7, 37423746, doi:10.1021/acs.jpclett.6b01501 (2016).

39 Baldini, E. et al. Strongly bound excitons in anatase TiO2 single crystals and nanoparticles. Nature Communications 8, 13, doi:10.1038/s41467-017-00016-6 (2017). 
Baldini, E. et al. Exciton control in a room temperature bulk semiconductor with coherent strain pulses. Science Advances 5, eaax2937, doi:10.1126/sciadv.aax2937 (2019).

41 Benson, E., Fortin, E. \& Mysyrowicz, A. Study of Anomalous Excitonic Transport in Cu2O. physica status solidi (b) 191, 345-367, doi:10.1002/pssb.2221910211 (1995).

42 Omelchenko, S. T., Tolstova, Y., Atwater, H. A. \& Lewis, N. S. Excitonic Effects in Emerging Photovoltaic Materials: A Case Study in Cu2O. ACS Energy Letters, 431-437, doi:10.1021/acsenergylett.6b00704 (2017).

43 Abdi, F. F. \& van de Krol, R. Nature and Light Dependence of Bulk Recombination in Co-PiCatalyzed BiVO4 Photoanodes. The Journal of Physical Chemistry C 116, 9398-9404, doi:10.1021/jp3007552 (2012).

44 Wang, G., Ling, Y. \& Li, Y. Oxygen-deficient metal oxide nanostructures for photoelectrochemical water oxidation and other applications. Nanoscale 4, 6682-6691, doi:10.1039/C2NR32222F (2012).

45 Segev, G. et al. High Solar Flux Concentration Water Splitting with Hematite ( $\alpha$-Fe2O3) Photoanodes. Advanced Energy Materials 6, 1500817, doi:10.1002/aenm.201500817 (2016).

46 Bassi, P. S. et al. Crystalline Fe2O3/Fe2TiO5 heterojunction nanorods with efficient charge separation and hole injection as photoanode for solar water oxidation. Nano Energy 22, 310318, doi:https://doi.org/10.1016/j.nanoen.2016.02.013 (2016).

47 Katoh, R., Murai, M. \& Furube, A. Electron-hole recombination in the bulk of a rutile TiO2 single crystal studied by sub-nanosecond transient absorption spectroscopy. Chemical Physics Letters 461, 238-241, doi:https://doi.org/10.1016/j.cplett.2008.07.021 (2008). Davies, D. W. et al. Descriptors for Electron and Hole Charge Carriers in Metal Oxides. The Journal of Physical Chemistry Letters 11, 438-444, doi:10.1021/acs.jpclett.9b03398 (2020). Inoue, Y. Photocatalytic water splitting by RuO2-loaded metal oxides and nitrides with d0and d10 -related electronic configurations. Energy \& Environmental Science 2, 364-386, doi:10.1039/B816677N (2009).

50 Sato, J., Kobayashi, H., Saito, N., Nishiyama, H. \& Inoue, Y. Photocatalytic activities for water decomposition of RuO2-loaded AlnO2 ( $\mathrm{A}=\mathrm{Li}, \mathrm{Na})$ with d10 configuration. Journal of Photochemistry and Photobiology A: Chemistry 158, 139-144, doi:https://doi.org/10.1016/S1010-6030(03)00028-5 (2003).

51 Walsh, A., Sokol, A. A., Buckeridge, J., Scanlon, D. O. \& Catlow, C. R. A. (ACS Publications, 2017).

52 Wang, J. et al. Stabilizing the oxygen vacancies and promoting water-oxidation kinetics in cobalt oxides by lower valence-state doping. Nano Energy 53, 144-151, doi:https://doi.org/10.1016/j.nanoen.2018.08.022 (2018).

53 Ruhle, S. et al. All-Oxide Photovoltaics. J. Phys. Chem. Lett. 3, 3755-3764, doi:10.1021/jz3017039 (2012).

54 Turner, G. M., Beard, M. C. \& Schmuttenmaer, C. A. Carrier Localization and Cooling in DyeSensitized Nanocrystalline Titanium Dioxide. The Journal of Physical Chemistry B 106, 1171611719, doi:10.1021/jp025844e (2002).

55 Jiang, C.-M. et al. Characterization of Photo-Induced Charge Transfer and Hot Carrier Relaxation Pathways in Spinel Cobalt Oxide (Co3O4). The Journal of Physical Chemistry C 118, 22774-22784, doi:10.1021/jp5071133 (2014).

56 Husek, J., Cirri, A., Biswas, S. \& Baker, L. R. Surface electron dynamics in hematite ( $\alpha$-Fe2O3): correlation between ultrafast surface electron trapping and small polaron formation. Chemical Science 8, 8170-8178, doi:10.1039/C7SC02826A (2017).

57 Ziwritsch, M. et al. Direct Time-Resolved Observation of Carrier Trapping and Polaron Conductivity in BiVO4. ACS Energy Letters 1, 888-894, doi:10.1021/acsenergylett.6b00423 (2016). 

dimensional liquid of Fröhlich polarons at the bare SrTiO3 surface. Nature Communications 6, 8585, doi:10.1038/ncomms9585 (2015). Santomauro, F. G. et al. Femtosecond X-ray absorption study of electron localization in photoexcited anatase TiO2. Scientific Reports 5, 14834, doi:10.1038/srep14834 (2015). Carneiro, L. M. et al. Excitation-wavelength-dependent small polaron trapping of photoexcited carriers in $\alpha$-Fe2O3. Nature Materials 16, 819-825, doi:10.1038/nmat4936 (2017).

61 Selim, S. et al. Impact of Oxygen Vacancy Occupancy on Charge Carrier Dynamics in BiVO4 Photoanodes. Journal of the American Chemical Society 141, 18791-18798, doi:10.1021/jacs.9b09056 (2019).

62 Greiner, M. T., Chai, L., Helander, M. G., Tang, W.-M. \& Lu, Z.-H. Transition Metal Oxide Work Functions: The Influence of Cation Oxidation State and Oxygen Vacancies. Advanced Functional Materials 22, 4557-4568, doi:10.1002/adfm.201200615 (2012). Gerosa, M., Gygi, F., Govoni, M. \& Galli, G. The role of defects and excess surface charges at finite temperature for optimizing oxide photoabsorbers. Nature Materials 17, 1122-1127, doi:10.1038/s41563-018-0192-4 (2018).

64 Emin, D., Seager, C. H. \& Quinn, R. K. Small-Polaron Hopping Motion in Some Chalcogenide Glasses. Physical Review Letters 28, 813-816, doi:10.1103/PhysRevLett.28.813 (1972).

65 Rettie, A. J. E., Chemelewski, W. D., Emin, D. \& Mullins, C. B. Unravelling Small-Polaron Transport in Metal Oxide Photoelectrodes. The Journal of Physical Chemistry Letters 7, 471479, doi:10.1021/acs.jpclett.5b02143 (2016). $\mathrm{Li}$, J. \& Wu, N. Semiconductor-based photocatalysts and photoelectrochemical cells for solar fuel generation: a review. Catal. Sci. Technol. 5, 1360-1384, doi:10.1039/c4cy00974f (2015). Makino, T., Segawa, Y., Tsukazaki, A., Ohtomo, A. \& Kawasaki, M. Electron transport in ZnO thin films. Applied Physics Letters 87, 022101, doi:10.1063/1.1991994 (2005). Yang, L. et al. Mo6+ Doped BiVO4 with improved Charge Separation and Oxidation Kinetics for Photoelectrochemical Water Splitting. Electrochimica Acta 256, 268-277, doi:https://doi.org/10.1016/i.electacta.2017.09.186 (2017). Cesar, I., Sivula, K., Kay, A., Zboril, R. \& Grätzel, M. Influence of Feature Size, Film Thickness, and Silicon Doping on the Performance of Nanostructured Hematite Photoanodes for Solar Water Splitting. The Journal of Physical Chemistry C 113, 772-782, doi:10.1021/jp809060p (2009).

70 Murthy, D. H. K. et al. Revealing the role of the Rh valence state, La doping level and Ru cocatalyst in determining the $\mathrm{H} 2$ evolution efficiency in doped SrTiO3 photocatalysts. Sustainable Energy \& Fuels 3, 208-218, doi:10.1039/C8SE00487K (2019).

71 Yamakata, A., Kawaguchi, M., Murachi, R., Okawa, M. \& Kamiya, I. Dynamics of Photogenerated Charge Carriers on Ni- and Ta-Doped SrTiO3 Photocatalysts Studied by Time-Resolved Absorption and Emission Spectroscopy. The Journal of Physical Chemistry $C$ 120, 7997-8004, doi:10.1021/acs.jpcc.6b01494 (2016).

72 Godin, R., Wang, Y., Zwijnenburg, M. A., Tang, J. \& Durrant, J. R. Time-Resolved Spectroscopic Investigation of Charge Trapping in Carbon Nitrides Photocatalysts for Hydrogen Generation. Journal of the American Chemical Society 139, 5216-5224, doi:10.1021/jacs.7b01547 (2017).

73 Tang, J., Cowan, A. J., Durrant, J. R. \& Klug, D. R. Mechanism of O2 Production from Water Splitting: Nature of Charge Carriers in Nitrogen Doped Nanocrystalline TiO2 Films and Factors Limiting O2 Production. The Journal of Physical Chemistry C 115, 3143-3150, doi:10.1021/jp1080093 (2011).

74 Barroso, M., Pendlebury, S. R., Cowan, A. J. \& Durrant, J. R. Charge carrier trapping, recombination and transfer in hematite (a-Fe2O3) water splitting photoanodes. Chemical Science 4, 2724-2734, doi:10.1039/C3SC50496D (2013). 
75 Yang, W. et al. Electron Accumulation Induces Efficiency Bottleneck for Hydrogen Production in Carbon Nitride Photocatalysts. Journal of the American Chemical Society 141, 1121911229, doi:10.1021/jacs.9b04556 (2019).

76 Steier, L. et al. Understanding the Role of Underlayers and Overlayers in Thin Film Hematite Photoanodes. Advanced Functional Materials 24, 7681-7688, doi:10.1002/adfm.201402742 (2014).

77 Zhang, Z., Nagashima, H. \& Tachikawa, T. Ultra-Narrow Depletion Layers in a Hematite Mesocrystal-Based Photoanode for Boosting Multihole Water Oxidation. Angew Chem Int Ed Engl 59, 9047-9054, doi:10.1002/anie.202001919 (2020).

$78 \mathrm{Li}$, J. et al. Photoelectrochemical performance enhanced by a nickel oxide-hematite $\mathrm{p}-\mathrm{n}$ junction photoanode. Chemical Communications 48, 8213-8215, doi:10.1039/C2CC30376K (2012).

79 Meng, X. Y. et al. Enhanced photoelectrochemical activity for $\mathrm{Cu}$ and Ti doped hematite: The first principles calculations. Applied Physics Letters 98, 112104, doi:10.1063/1.3567766 (2011).

80 Zandi, O. \& Hamann, T. W. The potential versus current state of water splitting with hematite. Physical Chemistry Chemical Physics 17, 22485-22503, doi:10.1039/C5CP04267D (2015).

81 Abdi, F. F. et al. Efficient solar water splitting by enhanced charge separation in a bismuth vanadate-silicon tandem photoelectrode. Nature Communications 4, 2195, doi:10.1038/ncomms3195 (2013).

82 Moss, B. et al. Linking in situ charge accumulation to electronic structure in doped SrTiO3 reveals design principles for hydrogen-evolving photocatalysts. Nature Materials, doi:10.1038/s41563-020-00868-2 (2021).

83 Steier, L. et al. Low-Temperature Atomic Layer Deposition of Crystalline and Photoactive Ultrathin Hematite Films for Solar Water Splitting. ACS Nano 9, 11775-11783, doi:10.1021/acsnano.5b03694 (2015).

84 Rettie, A. J. E. et al. Combined Charge Carrier Transport and Photoelectrochemical Characterization of BiVO4 Single Crystals: Intrinsic Behavior of a Complex Metal Oxide. Journal of the American Chemical Society 135, 11389-11396, doi:10.1021/ja405550k (2013).

85 Seabold, J. A., Zhu, K. \& Neale, N. R. Efficient solar photoelectrolysis by nanoporous Mo:BiVO4 through controlled electron transport. Physical Chemistry Chemical Physics 16, 1121-1131, doi:10.1039/C3CP54356K (2014).

86 Abdi, F. F., Savenije, T. J., May, M. M., Dam, B. \& van de Krol, R. The Origin of Slow Carrier Transport in BiVO4 Thin Film Photoanodes: A Time-Resolved Microwave Conductivity Study. The Journal of Physical Chemistry Letters 4, 2752-2757, doi:10.1021/jz4013257 (2013).

87 Ravensbergen, J. et al. Unraveling the Carrier Dynamics of BiVO4: A Femtosecond to Microsecond Transient Absorption Study. The Journal of Physical Chemistry C 118, 2779327800, doi:10.1021/jp509930s (2014).

88 Grigioni, I., Stamplecoskie, K. G., Selli, E. \& Kamat, P. V. Dynamics of Photogenerated Charge Carriers in WO3/BiVO4 Heterojunction Photoanodes. The Journal of Physical Chemistry C 119, 20792-20800, doi:10.1021/acs.jpcc.5b05128 (2015).

89 Butler, K. T. et al. Ultrafast carrier dynamics in BiVO4 thin film photoanode material: interplay between free carriers, trapped carriers and low-frequency lattice vibrations. Journal of Materials Chemistry A 4, 18516-18523, doi:10.1039/C6TA07177E (2016).

90 Moss, B. et al. Anisotropic Electron Transport Limits Performance of Bi2WO6 Photoanodes. The Journal of Physical Chemistry C 124, 18859-18867, doi:10.1021/acs.jpcc.0c03539 (2020).

91 Zhu, J. et al. Direct imaging of highly anisotropic photogenerated charge separations on different facets of a single BiVO4 photocatalyst. Angewandte Chemie 127, 9239-9242 (2015). 

and future needs: A mini-review. Electrochemistry Communications 63, 10-17, doi:http://dx.doi.org/10.1016/j.elecom.2015.12.003 (2016).

93 Lee, M. G. et al. Solution-processed metal oxide thin film nanostructures for water splitting photoelectrodes: a review. Journal of the Korean Ceramic Society 55, 185-202 (2018). Concina, I., Ibupoto, Z. H. \& Vomiero, A. Semiconducting metal oxide nanostructures for water splitting and photovoltaics. Advanced Energy Materials 7, 1700706 (2017). Zhang, W. \& Zhou, K. Ultrathin Two-Dimensional Nanostructured Materials for Highly Efficient Water Oxidation. Small 13, 1700806 (2017).

96 Tamirat, A. G., Rick, J., Dubale, A. A., Su, W.-N. \& Hwang, B.-J. Using hematite for photoelectrochemical water splitting: a review of current progress and challenges. Nanoscale Horizons 1, 243-267, doi:10.1039/C5NH00098J (2016).

97 Brillet, J. et al. Highly efficient water splitting by a dual-absorber tandem cell. Nature Photonics 6, 824-828, doi:10.1038/nphoton.2012.265 (2012).

98 Cherepy, N. J., Liston, D. B., Lovejoy, J. A., Deng, H. \& Zhang, J. Z. Ultrafast Studies of Photoexcited Electron Dynamics in $\gamma$ - and $\alpha-\mathrm{Fe} 2 \mathrm{O} 3$ Semiconductor Nanoparticles. The Journal of Physical Chemistry B 102, 770-776, doi:10.1021/jp973149e (1998). Photoanodes under Applied Bias. Journal of the American Chemical Society 136, 9854-9857, doi:10.1021/ja504473e (2014).

100 Lyu, H. et al. An Al-doped SrTiO 3 photocatalyst maintaining sunlight-driven overall water splitting activity for over $1000 \mathrm{~h}$ of constant illumination. Chemical science 10, 3196-3201 (2019).

101 Ham, Y. et al. Flux-mediated doping of SrTiO 3 photocatalysts for efficient overall water splitting. Journal of Materials Chemistry A 4, 3027-3033 (2016).

102 Takata, T. et al. Photocatalytic water splitting with a quantum efficiency of almost unity. Nature 581, 411-414, doi:10.1038/s41586-020-2278-9 (2020).

103 Barroso, M. et al. Dynamics of photogenerated holes in surface modified $\alpha$ $\mathrm{Fe}<$ sub $>2</$ sub $>0<$ sub $>3</$ sub $>$ photoanodes for solar water splitting. Proceedings of the National Academy of Sciences 109, 15640-15645, doi:10.1073/pnas.1118326109 (2012).

104 Klahr, B., Gimenez, S., Fabregat-Santiago, F., Bisquert, J. \& Hamann, T. W. Electrochemical and photoelectrochemical investigation of water oxidation with hematite electrodes. Energy \& Environmental Science 5, 7626-7636, doi:10.1039/C2EE21414H (2012).

105 Gerischer, H. Electrochemical photo and solar cells principles and some experiments. Journal of Electroanalytical Chemistry and Interfacial Electrochemistry 58, 263-274, doi:https://doi.org/10.1016/S0022-0728(75)80359-7 (1975).

106 Gerischer, H. Electrochemical Behavior of Semiconductors under Illumination. Journal of The Electrochemical Society 113, 1174, doi:10.1149/1.2423779 (1966).

107 Ma, Y., Pendlebury, S. R., Reynal, A., Le Formal, F. \& Durrant, J. R. Dynamics of photogenerated holes in undoped BiVO4 photoanodes for solar water oxidation. Chemical Science 5, 2964, doi:10.1039/c4sc00469h (2014).

108 Cowan, A. J., Tang, J., Leng, W., Durrant, J. R. \& Klug, D. R. Water Splitting by Nanocrystalline $\mathrm{TiO} 2$ in a Complete Photoelectrochemical Cell Exhibits Efficiencies Limited by Charge Recombination. The Journal of Physical Chemistry C 114, 4208-4214, doi:10.1021/jp909993w (2010).

109 Upul Wijayantha, K. G., Saremi-Yarahmadi, S. \& Peter, L. M. Kinetics of oxygen evolution at $\alpha$-Fe2O3 photoanodes: a study by photoelectrochemical impedance spectroscopy. Physical Chemistry Chemical Physics 13, 5264-5270, doi:10.1039/C0CP02408B (2011).

110 Sivula, K., Le Formal, F. \& Grätzel, M. Solar Water Splitting: Progress Using Hematite ( $\alpha$ Fe2O3) Photoelectrodes. ChemSusChem 4, 432-449, doi:10.1002/cssc.201000416 (2011). 
111 Pendlebury, S. R. et al. Correlating long-lived photogenerated hole populations with photocurrent densities in hematite water oxidation photoanodes. Energy \& Environmental Science 5, 6304-6312, doi:10.1039/C1EE02567H (2012).

112 Kim, T. W. \& Choi, K.-S. Nanoporous $\mathrm{BiVO}_{4}$ Photoanodes with Dual-Layer Oxygen Evolution Catalysts for Solar Water Splitting. Science 343, 990-994 (2014).

113 Ma, Y. et al. Rate Law Analysis of Water Oxidation and Hole Scavenging on a BiVO4 Photoanode. ACS Energy Letters 1, 618-623, doi:10.1021/acsenergylett.6b00263 (2016).

114 Dotan, H., Sivula, K., Gratzel, M., Rothschild, A. \& Warren, S. C. Probing the photoelectrochemical properties of hematite ([small alpha]-Fe2O3) electrodes using hydrogen peroxide as a hole scavenger. Energy \& Environmental Science 4, 958-964, doi:10.1039/COEE00570C (2011).

115 Chen, X., Aschaffenburg, D. \& Cuk, T. One-electron intermediates of water oxidation \& the role of solvation in their stability. Journal of Materials Chemistry A 5, 11410-11417, doi:10.1039/C7TA02240A (2017).

116 Chen, X. et al. The Formation Time of $\mathrm{Ti}-\mathrm{O} \bullet$ and $\mathrm{Ti}-\mathrm{O} \bullet-\mathrm{Ti}$ Radicals at the n-SrTiO3/Aqueous Interface during Photocatalytic Water Oxidation. Journal of the American Chemical Society 139, 1830-1841, doi:10.1021/jacs.6b09550 (2017).

117 Herlihy, D. M. et al. Detecting the oxyl radical of photocatalytic water oxidation at an nSrTiO3/aqueous interface through its subsurface vibration. Nature Chemistry 8, 549-555, doi:10.1038/nchem.2497 (2016).

118 Ahmed, S., Leduc, J. \& Haller, S. Photoelectrochemical and impedance characteristics of specular hematite. 1. Photoelectrochemical parallel conductance, and trap rate studies. The Journal of Physical Chemistry 92, 6655-6660 (1988).

119 Glasscock, J. A., Barnes, P. R. F., Plumb, I. C. \& Savvides, N. Enhancement of Photoelectrochemical Hydrogen Production from Hematite Thin Films by the Introduction of Ti and Si. The Journal of Physical Chemistry C 111, 16477-16488, doi:10.1021/jp074556I (2007).

120 Le Formal, F., Sivula, K. \& Grätzel, M. The Transient Photocurrent and Photovoltage Behavior of a Hematite Photoanode under Working Conditions and the Influence of Surface Treatments. The Journal of Physical Chemistry C 116, 26707-26720, doi:10.1021/jp308591k (2012).

121 Le Formal, F. et al. Back electron-hole recombination in hematite photoanodes for water splitting. Journal of the American Chemical Society 136, 2564-2574, doi:10.1021/ja412058x (2014).

122 Ma, Y., Le Formal, F., Kafizas, A., Pendlebury, S. R. \& Durrant, J. R. Efficient suppression of back electron/hole recombination in cobalt phosphate surface-modified undoped bismuth vanadate photoanodes. Journal of Materials Chemistry A 3, 20649-20657, doi:10.1039/C5TA05826K (2015).

123 Kafizas, A., Godin, R. \& Durrant, J. R. in Semiconductors and Semimetals Vol. 97 (eds Zetian Mi, Lianzhou Wang, \& Chennupati Jagadish) 3-46 (Elsevier, 2017).

124 Peter, L. M., Wijayantha, K. G. U. \& Tahir, A. A. Kinetics of light-driven oxygen evolution at $\alpha$ Fe2O3 electrodes. Faraday Discussions 155, 309-322, doi:10.1039/C1FD00079A (2012).

125 Kafizas, A. et al. Water Oxidation Kinetics of Accumulated Holes on the Surface of a TiO2 Photoanode: A Rate Law Analysis. ACS Catalysis 7, 4896-4903, doi:10.1021/acscatal.7b01150 (2017).

126 Zandi, O. \& Hamann, T. W. Enhanced Water Splitting Efficiency Through Selective Surface State Removal. The Journal of Physical Chemistry Letters 5, 1522-1526, doi:10.1021/jz500535a (2014).

127 Liu, R., Zheng, Z., Spurgeon, J. \& Yang, X. Enhanced photoelectrochemical water-splitting performance of semiconductors by surface passivation layers. Energy \& Environmental Science 7, 2504-2517, doi:10.1039/C4EE00450G (2014). 
128 Zhang, P., Wang, T. \& Gong, J. Passivation of surface states by ALD-grown TiO2 overlayers on Ta3N5 anodes for photoelectrochemical water oxidation. Chemical Communications 52, 8806-8809, doi:10.1039/C6CC03411J (2016).

129 Le Formal, F. et al. Passivating surface states on water splitting hematite photoanodes with alumina overlayers. Chemical Science 2, 737-743, doi:10.1039/COSC00578A (2011).

130 Ding, T. X., Olshansky, J. H., Leone, S. R. \& Alivisatos, A. P. Efficiency of Hole Transfer from Photoexcited Quantum Dots to Covalently Linked Molecular Species. Journal of the American Chemical Society 137, 2021-2029, doi:10.1021/ja512278a (2015).

131 Olshansky, J. H., Ding, T. X., Lee, Y. V., Leone, S. R. \& Alivisatos, A. P. Hole Transfer from Photoexcited Quantum Dots: The Relationship between Driving Force and Rate. Journal of the American Chemical Society 137, 15567-15575, doi:10.1021/jacs.5b10856 (2015).

132 Smith, A. M. \& Nie, S. Semiconductor Nanocrystals: Structure, Properties, and Band Gap Engineering. Accounts of Chemical Research 43, 190-200, doi:10.1021/ar9001069 (2010).

133 Zawadzki, P., Laursen, A. B., Jacobsen, K. W., Dahl, S. \& Rossmeisl, J. Oxidative trends of TiO2-hole trapping at anatase and rutile surfaces. Energy \& Environmental Science 5, 98669869, doi:10.1039/C2EE22721E (2012).

134 Klahr, B., Gimenez, S., Fabregat-Santiago, F., Hamann, T. \& Bisquert, J. Water oxidation at hematite photoelectrodes: the role of surface states. Journal of the American Chemical Society 134, 4294-4302, doi:10.1021/ja210755h (2012).

135 Shi, Q. et al. Role of Tungsten Doping on the Surface States in BiVO4 Photoanodes for Water Oxidation: Tuning the Electron Trapping Process. ACS Catalysis 8, 3331-3342, doi:10.1021/acscatal.7b04277 (2018).

136 Linsebigler, A. L., Lu, G. \& Yates, J. T. Photocatalysis on TiO2 Surfaces: Principles, Mechanisms, and Selected Results. Chemical Reviews 95, 735-758, doi:10.1021/cr00035a013 (1995).

137 Sudhagar, P., Devadoss, A., Nakata, K., Terashima, C. \& Fujishima, A. Enhanced Photoelectrocatalytic Water Splitting at Hierarchical Gd3+:TiO2Nanostructures through Amplifying Light Reception and Surface States Passivation. Journal of The Electrochemical Society 162, H108-H114, doi:10.1149/2.0161503jes (2014).

138 Peter, L. M. Dynamic aspects of semiconductor photoelectrochemistry. Chemical Reviews 90, 753-769, doi:10.1021/cr00103a005 (1990).

139 Colombo, D. P. \& Bowman, R. M. Femtosecond diffuse reflectance spectroscopy of TiO2 powders. The Journal of Physical Chemistry 99, 11752-11756, doi:10.1021/j100030a020 (1995).

140 Leng, W. H., Zhang, Z., Zhang, J. Q. \& Cao, C. N. Investigation of the Kinetics of a TiO2 Photoelectrocatalytic Reaction Involving Charge Transfer and Recombination through Surface States by Electrochemical Impedance Spectroscopy. The Journal of Physical Chemistry B 109, 15008-15023, doi:10.1021/jp051821z (2005).

141 Gomes, W. P. \& Vanmaekelbergh, D. Impedance spectroscopy at semiconductor electrodes: Review and recent developments. Electrochimica Acta 41, 967-973, doi:https://doi.org/10.1016/0013-4686(95)00427-0 (1996).

142 Gomes, W. P. \& Cardon, F. Surface States at the Single Crystal Zinc Oxide/Electrolyte Interface. I. Impedance Measurements. Berichte der Bunsengesellschaft für physikalische Chemie 74, 431-436, doi:10.1002/bbpc.19700740503 (1970).

143 Cardon, F. Impedance-and noise-spectrum calculation for a semiconductor-electrolyte interface with electron transfer through surface states. Physica 57, 390-396 (1972).

144 Bertoluzzi, L., Lopez-Varo, P., Jiménez Tejada, J. A. \& Bisquert, J. Charge transfer processes at the semiconductor/electrolyte interface for solar fuel production: insight from impedance spectroscopy. Journal of Materials Chemistry A 4, 2873-2879, doi:10.1039/C5TA03210E (2016). 
145 Klahr, B. \& Hamann, T. Water Oxidation on Hematite Photoelectrodes: Insight into the Nature of Surface States through In Situ Spectroelectrochemistry. The Journal of Physical Chemistry C 118, 10393-10399, doi:10.1021/jp500543z (2014).

146 Wang, X. et al. Transient Absorption Spectroscopy of Anatase and Rutile: The Impact of Morphology and Phase on Photocatalytic Activity. The Journal of Physical Chemistry C 119, 10439-10447, doi:10.1021/acs.jpcc.5b01858 (2015).

147 Gao, Y. \& Hamann, T. W. Elucidation of CuWO4 Surface States During Photoelectrochemical Water Oxidation. The Journal of Physical Chemistry Letters 8, 2700-2704, doi:10.1021/acs.jpclett.7b00664 (2017).

148 Morris, M. R., Pendlebury, S. R., Hong, J., Dunn, S. \& Durrant, J. R. Effect of Internal Electric Fields on Charge Carrier Dynamics in a Ferroelectric Material for Solar Energy Conversion. Advanced Materials 28, 7123-7128, doi:10.1002/adma.201601238 (2016).

$149 \mathrm{Mu}$, L. et al. Enhancing charge separation on high symmetry SrTiO3 exposed with anisotropic facets for photocatalytic water splitting. Energy \& Environmental Science 9, 2463-2469, doi:10.1039/C6EE00526H (2016).

150 Zhang, J., Xu, Q., Feng, Z., Li, M. \& Li, C. Importance of the relationship between surface phases and photocatalytic activity of TiO2. Angewandte Chemie 120, 1790-1793 (2008).

151 Li, R. et al. Spatial separation of photogenerated electrons and holes among $\{010\}$ and $\{110\}$ crystal facets of BiVO4. Nature Communications 4, 1432, doi:10.1038/ncomms2401 (2013).

152 Liu, L. et al. Graphdiyne Derivative as Multifunctional Solid Additive in Binary Organic Solar Cells with 17.3\% Efficiency and High Reproductivity. Advanced Materials 32, 1907604, doi:https://doi.org/10.1002/adma.201907604 (2020).

153 Liu, Y. et al. Internal-Field-Enhanced Charge Separation in a Single-Domain Ferroelectric PbTiO(3) Photocatalyst. Adv Mater 32, e1906513, doi:10.1002/adma.201906513 (2020).

154 Li, Z. et al. Surface-Polarity-Induced Spatial Charge Separation Boosts Photocatalytic Overall Water Splitting on GaN Nanorod Arrays. Angewandte Chemie International Edition 59, 935942, doi:https://doi.org/10.1002/anie.201912844 (2020).

155 Lin, Y. et al. Growth of p-Type Hematite by Atomic Layer Deposition and Its Utilization for Improved Solar Water Splitting. Journal of the American Chemical Society 134, 5508-5511, doi:10.1021/ja300319g (2012).

156 Ahmed, M. G. et al. A Facile Surface Passivation of Hematite Photoanodes with TiO2 Overlayers for Efficient Solar Water Splitting. ACS Applied Materials \& Interfaces 7, 2405324062, doi:10.1021/acsami.5b07065 (2015).

157 Hisatomi, T. et al. Cathodic shift in onset potential of solar oxygen evolution on hematite by 13-group oxide overlayers. Energy \& Environmental Science 4, 2512-2515, doi:10.1039/C1EE01194D (2011).

158 Scanlon, D. O. et al. Band alignment of rutile and anatase TiO2. Nature Materials 12, 798801, doi:10.1038/nmat3697 (2013).

159 Kafizas, A. et al. Where Do Photogenerated Holes Go in Anatase:Rutile TiO2? A Transient Absorption Spectroscopy Study of Charge Transfer and Lifetime. The Journal of Physical Chemistry A 120, 715-723, doi:10.1021/acs.jpca.5b11567 (2016).

$160 \mathrm{Li}, \mathrm{A}$. et al. Understanding the anatase-rutile phase junction in charge separation and transfer in a TiO2 electrode for photoelectrochemical water splitting. Chemical Science 7, 6076-6082, doi:10.1039/C6SC01611A (2016).

161 Selim, S. et al. WO3/BiVO4: impact of charge separation at the timescale of water oxidation. Chemical Science 10, 2643-2652, doi:10.1039/C8SC04679D (2019).

162 Chae, S. Y. et al. Insight into charge separation in WO3/BiVO4 heterojunction for solar water splitting. ACS Applied Materials \& Interfaces 9, 19780-19790 (2017).

163 Kalanoor, B. S., Seo, H. \& Kalanur, S. S. Recent developments in photoelectrochemical watersplitting using WO3/BiVO4 heterojunction photoanode: A review. Materials Science for Energy Technologies 1, 49-62 (2018). 
164 Grigioni, I. et al. In Operando Photoelectrochemical Femtosecond Transient Absorption Spectroscopy of WO3/BiVO4 Heterojunctions. ACS Energy Letters 4, 2213-2219, doi:10.1021/acsenergylett.9b01150 (2019).

$165 \mathrm{Ye}, \mathrm{K} .-\mathrm{H}$. et al. Enhancing photoelectrochemical water splitting by combining work function tuning and heterojunction engineering. Nature Communications 10, 3687, doi:10.1038/s41467-019-11586-y (2019).

166 Seabold, J. A. \& Choi, K.-S. Effect of a Cobalt-Based Oxygen Evolution Catalyst on the Stability and the Selectivity of Photo-Oxidation Reactions of a WO3 Photoanode. Chemistry of Materials 23, 1105-1112, doi:10.1021/cm1019469 (2011).

167 Zhong, D. K. \& Gamelin, D. R. Photoelectrochemical Water Oxidation by Cobalt Catalyst ("Co-Pi")/ $\alpha-\mathrm{Fe} 2 \mathrm{O} 3$ Composite Photoanodes: Oxygen Evolution and Resolution of a Kinetic Bottleneck. Journal of the American Chemical Society 132, 4202-4207, doi:10.1021/ja908730h (2010).

168 Lin, F. \& Boettcher, S. W. Adaptive semiconductor/electrocatalyst junctions in water-splitting photoanodes. Nature Materials 13, 81-86, doi:10.1038/nmat3811 (2014).

169 Barroso, M. et al. The Role of Cobalt Phosphate in Enhancing the Photocatalytic Activity of $\alpha$ Fe2O3 toward Water Oxidation. Journal of the American Chemical Society 133, 14868-14871, doi:10.1021/ja205325v (2011).

170 Peter, L. M. Energetics and kinetics of light-driven oxygen evolution at semiconductor electrodes: the example of hematite. Journal of Solid State Electrochemistry 17, 315-326, doi:10.1007/s10008-012-1957-3 (2012).

171 Seabold, J. A. \& Choi, K.-S. Efficient and Stable Photo-Oxidation of Water by a Bismuth Vanadate Photoanode Coupled with an Iron Oxyhydroxide Oxygen Evolution Catalyst. Journal of the American Chemical Society 134, 2186-2192, doi:10.1021/ja209001d (2012).

172 Ye, H., Park, H. S. \& Bard, A. J. Screening of Electrocatalysts for Photoelectrochemical Water Oxidation on W-Doped BiVO4 Photocatalysts by Scanning Electrochemical Microscopy. The Journal of Physical Chemistry C 115, 12464-12470, doi:10.1021/jp200852c (2011).

173 Moss, B. et al. Unraveling Charge Transfer in CoFe Prussian Blue Modified BiVO4 Photoanodes. ACS Energy Letters 4, 337-342, doi:10.1021/acsenergylett.8b02225 (2019).

174 Mills, T. J., Lin, F. \& Boettcher, S. W. Theory and Simulations of Electrocatalyst-Coated Semiconductor Electrodes for Solar Water Splitting. Physical Review Letters 112, 148304, doi:10.1103/PhysRevLett.112.148304 (2014).

175 Mi, Q., Zhanaidarova, A., Brunschwig, B. S., Gray, H. B. \& Lewis, N. S. A quantitative assessment of the competition between water and anion oxidation at WO3 photoanodes in acidic aqueous electrolytes. Energy \& Environmental Science 5, 5694-5700, doi:10.1039/C2EE02929D (2012).

176 Hill, J. C. \& Choi, K.-S. Effect of Electrolytes on the Selectivity and Stability of n-type WO3 Photoelectrodes for Use in Solar Water Oxidation. The Journal of Physical Chemistry C 116, 7612-7620, doi:10.1021/jp209909b (2012).

177 Santato, C., Ulmann, M. \& Augustynski, J. Photoelectrochemical Properties of Nanostructured Tungsten Trioxide Films. The Journal of Physical Chemistry B 105, 936-940, doi:10.1021/jp002232q (2001).

178 Lhermitte, C. R., Garret Verwer, J. \& Bartlett, B. M. Improving the stability and selectivity for the oxygen-evolution reaction on semiconducting WO3photoelectrodes with a solid-state FeOOH catalyst. J. Mater. Chem. A 4, 2960-2968, doi:10.1039/c5ta04747a (2016).

179 Klepser, B. M. \& Bartlett, B. M. Anchoring a Molecular Iron Catalyst to Solar-Responsive WO3 Improves the Rate and Selectivity of Photoelectrochemical Water Oxidation. Journal of the American Chemical Society 136, 1694-1697, doi:10.1021/ja4086808 (2014).

180 Grigioni, I. et al. Wavelength-Dependent Ultrafast Charge Carrier Separation in the WO3/BiVO4 Coupled System. ACS Energy Letters 2, 1362-1367, doi:10.1021/acsenergylett.7b00216 (2017). 
181 Kim, W. et al. Promoting water photooxidation on transparent WO3 thin films using an alumina overlayer. Energy \& Environmental Science 6, 3732, doi:10.1039/c3ee42151a (2013).

$182 \mathrm{Li}, \mathrm{W}$. et al. Comparison of heterogenized molecular and heterogeneous oxide catalysts for photoelectrochemical water oxidation. Energy \& Environmental Science 9, 1794-1802, doi:10.1039/C5EE03871E (2016).

183 Tang, J., Durrant, J. R. \& Klug, D. R. Mechanism of Photocatalytic Water Splitting in TiO2. Reaction of Water with Photoholes, Importance of Charge Carrier Dynamics, and Evidence for Four-Hole Chemistry. Journal of the American Chemical Society 130, 13885-13891, doi:10.1021/ja8034637 (2008).

184 Cowan, A. J. et al. Activation Energies for the Rate-Limiting Step in Water Photooxidation by Nanostructured $\alpha-\mathrm{Fe} 2 \mathrm{O} 3$ and TiO2. Journal of the American Chemical Society 133, 1013410140, doi:10.1021/ja200800t (2011).

185 Mesa, C. A. et al. Multihole water oxidation catalysis on haematite photoanodes revealed by operando spectroelectrochemistry and DFT. Nature Chemistry 12, 82-89, doi:10.1038/s41557-019-0347-1 (2020).

186 Berardi, S. et al. Molecular artificial photosynthesis. Chemical Society Reviews 43, 7501-7519 (2014).

187 Kuo, D.-Y. et al. Influence of Surface Adsorption on the Oxygen Evolution Reaction on IrO2(110). Journal of the American Chemical Society, doi:10.1021/jacs.6b11932 (2017).

188 Kuo, D.-Y. et al. Measurements of Oxygen Electroadsorption Energies and Oxygen Evolution Reaction on RuO2(110): A Discussion of the Sabatier Principle and Its Role in Electrocatalysis. Journal of the American Chemical Society 140, 17597-17605, doi:10.1021/jacs.8b09657 (2018).

189 Rao, R. R. et al. Towards identifying the active sites on RuO2(110) in catalyzing oxygen evolution. Energy \& Environmental Science 10, 2626-2637, doi:10.1039/C7EE02307C (2017).

190 Kuznetsov, D. A. et al. Tuning Redox Transitions via Inductive Effect in Metal Oxides and Complexes, and Implications in Oxygen Electrocatalysis. Joule 2, 225-244, doi:https://doi.org/10.1016/j.joule.2017.11.014 (2018).

191 Rossmeisl, J., Qu, Z. W., Zhu, H., Kroes, G. J. \& Nørskov, J. K. Electrolysis of water on oxide surfaces. Journal of Electroanalytical Chemistry 607, 83-89, doi:https://doi.org/10.1016/j.jelechem.2006.11.008 (2007).

192 Seh, Z. W. et al. Combining theory and experiment in electrocatalysis: Insights into materials design. Science 355, eaad4998, doi:10.1126/science.aad4998 (2017).

193 Man, I. C. et al. Universality in Oxygen Evolution Electrocatalysis on Oxide Surfaces. ChemCatChem 3, 1159-1165, doi:10.1002/cctc.201000397 (2011).

194 Montoya, J. H. et al. Materials for solar fuels and chemicals. Nature Materials 16, 70-81, doi:10.1038/nmat4778 (2017).

195 van Oversteeg, C. H. M., Doan, H. Q., de Groot, F. M. F. \& Cuk, T. In situ X-ray absorption spectroscopy of transition metal based water oxidation catalysts. Chemical Society Reviews 46, 102-125, doi:10.1039/C6CS00230G (2017).

196 Kanan, M. W. et al. Structure and Valency of a Cobalt-Phosphate Water Oxidation Catalyst Determined by in Situ X-ray Spectroscopy. Journal of the American Chemical Society 132, 13692-13701, doi:10.1021/ja1023767 (2010).

197 Gorlin, Y. et al. In Situ X-ray Absorption Spectroscopy Investigation of a Bifunctional Manganese Oxide Catalyst with High Activity for Electrochemical Water Oxidation and Oxygen Reduction. Journal of the American Chemical Society 135, 8525-8534, doi:10.1021/ja3104632 (2013).

198 Zheng, X. et al. Theory-driven design of high-valence metal sites for water oxidation confirmed using in situ soft X-ray absorption. Nature Chemistry 10, 149-154, doi:10.1038/nchem.2886 (2018). 
199 Joya, K. S. \& Sala, X. In situ Raman and surface-enhanced Raman spectroscopy on working electrodes: spectroelectrochemical characterization of water oxidation electrocatalysts. Physical Chemistry Chemical Physics 17, 21094-21103, doi:10.1039/C4CP05053C (2015).

200 Wang, D. et al. In Situ X-ray Absorption Near-Edge Structure Study of Advanced NiFe(OH)x Electrocatalyst on Carbon Paper for Water Oxidation. The Journal of Physical Chemistry C 119, 19573-19583, doi:10.1021/acs.jpcc.5b02685 (2015).

201 Lee, K. J., Elgrishi, N., Kandemir, B. \& Dempsey, J. L. Electrochemical and spectroscopic methods for evaluating molecular electrocatalysts. Nature Reviews Chemistry 1, 0039, doi:10.1038/s41570-017-0039 (2017).

202 Vojvodic, A. et al. On the behavior of Brønsted-Evans-Polanyi relations for transition metal oxides. The Journal of Chemical Physics 134, 244509, doi:10.1063/1.3602323 (2011).

203 Cheng, J. et al. Brønsted-Evans-Polanyi Relation of Multistep Reactions and Volcano Curve in Heterogeneous Catalysis. The Journal of Physical Chemistry C 112, 1308-1311, doi:10.1021/jp711191j (2008).

204 Li, Y., Tang, Z., Zhang, J. \& Zhang, Z. Enhanced photocatalytic performance of tungsten oxide through tuning exposed facets and introducing oxygen vacancies. Journal of Alloys and Compounds 708, 358-366, doi:http://doi.org/10.1016/i.jallcom.2017.03.046 (2017).

205 Yuan, H., Han, K., Dubbink, D., Mul, G. \& ten Elshof, J. E. Modulating the External Facets of Functional Nanocrystals Enabled by Two-Dimensional Oxide Crystal Templates. ACS Catalysis 7, 6858-6863, doi:10.1021/acscatal.7b02605 (2017).

206 Bhatt, M. D. \& Lee, J. S. Recent theoretical progress in the development of photoanode materials for solar water splitting photoelectrochemical cells. J. Mater. Chem. A 3, 1063210659, doi:10.1039/c5ta00257e (2015).

207 Wang, S. et al. Synergistic crystal facet engineering and structural control of WO3 films exhibiting unprecedented photoelectrochemical performance. Nano Energy 24, 94-102, doi:https://doi.org/10.1016/j.nanoen.2016.04.010 (2016).

208 Wang, D. et al. Crystal Facet Dependence of Water Oxidation on BiVO4 Sheets under Visible Light Irradiation. Chemistry - A European Journal 17, 1275-1282, doi:10.1002/chem.201001636 (2011).

209 Han, H. S. et al. Boosting the solar water oxidation performance of a BiVO4 photoanode by crystallographic orientation control. Energy \& Environmental Science 11, 1299-1306, doi:10.1039/C8EE00125A (2018).

210 Suntivich, J., Perry, E. E., Gasteiger, H. A. \& Shao-Horn, Y. The Influence of the Cation on the Oxygen Reduction and Evolution Activities of Oxide Surfaces in Alkaline Electrolyte. Electrocatalysis 4, 49-55, doi:10.1007/s12678-012-0118-x (2013).

211 Garcia, A. C., Touzalin, T., Nieuwland, C., Perini, N. \& Koper, M. T. M. Enhancement of Oxygen Evolution Activity of Nickel Oxyhydroxide by Electrolyte Alkali Cations. Angewandte Chemie International Edition 58, 12999-13003, doi:10.1002/anie.201905501 (2019).

212 Stoerzinger, K. A. et al. The Role of Ru Redox in pH-Dependent Oxygen Evolution on Rutile Ruthenium Dioxide Surfaces. Chem 2, 668-675, doi:https://doi.org/10.1016/j.chempr.2017.04.001 (2017).

213 Giordano, L. et al. pH dependence of OER activity of oxides: Current and future perspectives. Catalysis Today 262, 2-10, doi:https://doi.org/10.1016/i.cattod.2015.10.006 (2016).

214 Butler, M. A. Photoelectrolysis and physical properties of the semiconducting electrode $\mathrm{WO}_{3}$ Journal of Applied Physics 48, 1914-1920, doi:10.1063/1.323948 (1977).

215 Schulze, M., Kunz, V., Frischmann, P. D. \& Würthner, F. A supramolecular ruthenium macrocycle with high catalytic activity for water oxidation that mechanistically mimics photosystem II. Nature Chemistry 8, 576-583, doi:10.1038/nchem.2503 (2016).

216 Vinyard, D. J., Ananyev, G. M. \& Dismukes, G. C. Photosystem II: The Reaction Center of Oxygenic Photosynthesis. Annual Review of Biochemistry 82, 577-606, doi:10.1146/annurevbiochem-070511-100425 (2013). 
217 Francàs, L. et al. Spectroelectrochemical study of water oxidation on nickel and iron oxyhydroxide electrocatalysts. Nature Communications 10, 5208, doi:10.1038/s41467-01913061-0 (2019).

218 Takashima, T., Hashimoto, K. \& Nakamura, R. Mechanisms of pH-Dependent Activity for Water Oxidation to Molecular Oxygen by MnO2 Electrocatalysts. Journal of the American Chemical Society 134, 1519-1527, doi:10.1021/ja206511w (2012).

219 Ooka, H. et al. Legitimate intermediates of oxygen evolution on iridium oxide revealed by in situ electrochemical evanescent wave spectroscopy. Physical Chemistry Chemical Physics 18, 15199-15204, doi:10.1039/C6CP02385A (2016).

220 Allen, J. B. \& Larry, R. F. Electrochemical methods fundamentals and applications. (John Wiley \& Sons, 2001).

221 Bockris, J. O. M., Reddy, A. K. N. \& Gamboa-Aldeco, M. in Modern Electrochemistry 2A: Fundamentals of Electrodics (eds John O'M Bockris, Amulya K. N. Reddy, \& Maria GamboaAldeco) Ch. Electrodics, 1035-1400 (Springer US, 2000).

222 Lewis, N. S. Progress in Understanding Electron-Transfer Reactions at Semiconductor/Liquid Interfaces. The Journal of Physical Chemistry B 102, 4843-4855, doi:10.1021/jp9803586 (1998).

223 Rosenbluth, M. L. \& Lewis, N. S. " Ideal" behavior of the open circuit voltage of semiconductor/liquid junctions. The Journal of Physical Chemistry 93, 3735-3740 (1989).

224 Lewis, N. S. An analysis of charge transfer rate constants for semiconductor/liquid interfaces. Annual review of physical chemistry 42, 543-580 (1991).

225 Gärtner, W. W. Depletion-Layer Photoeffects in Semiconductors. Physical Review 116, 84-87, doi:10.1103/PhysRev.116.84 (1959).

226 Kautek, W., Gerischer, H. \& Tributsch, H. The Role of Carrier Diffusion and Indirect Optical Transitions in the Photoelectrochemical Behavior of Layer Type d-Band Semiconductors. Journal of The Electrochemical Society 127, 2471 (1980).

227 Beranek, R. Selectivity of Chemical Conversions: Do Light-Driven Photoelectrocatalytic Processes Hold Special Promise? Angewandte Chemie International Edition 58, 16724-16729 (2019).

228 Wilson, R. H. A model for the current-voltage curve of photoexcited semiconductor electrodes. Journal of Applied Physics 48, $4292-4297$ (1977).

229 Albery, W. J., Bartlett, P. N., Hamnett, A. \& Dare-Edwards, M. P. The transport and kinetics of minority carriers in illuminated semiconductor electrodes. Journal of The Electrochemical Society 128, 1492 (1981).

230 Reichman, J. The current-voltage characteristics of semiconductor-electrolyte junction photovoltaic cells. Applied Physics Letters 36, 574-577 (1980).

231 Reiss, H. Photocharacteristics for Electrolyte-Semiconductor Junctions. Journal of The Electrochemical Society 125, 937 (1978).

232 Pastor, E. et al. Spectroelectrochemical analysis of the mechanism of (photo)electrochemical hydrogen evolution at a catalytic interface. Nature Communications 8, 14280, doi:10.1038/ncomms14280 (2017).

233 Le Formal, F. et al. Rate law analysis of water oxidation on a hematite surface. J Am Chem Soc 137, 6629-6637, doi:10.1021/jacs.5b02576 (2015).

234 Soedergren, S., Hagfeldt, A., Olsson, J. \& Lindquist, S.-E. Theoretical Models for the Action Spectrum and the Current-Voltage Characteristics of Microporous Semiconductor Films in Photoelectrochemical Cells. The Journal of Physical Chemistry 98, 5552-5556, doi:10.1021/j100072a023 (1994).

235 Gerischer, H. The impact of semiconductors on the concepts of electrochemistry. Electrochimica Acta 35, 1677-1699 (1990).

236 Ahlgren, W. L. Analysis of the Current-Voltage Characteristics of Photoelectrolysis Cells. Journal of The Electrochemical Society 128, 2123 (1981). 
237 Lewis, N. S. Chemical Control of Charge Transfer and Recombination at Semiconductor Photoelectrode Surfaces. Inorganic Chemistry 44, 6900-6911, doi:10.1021/ic051118p (2005).

238 Lewis, N. S. Mechanistic studies of light-induced charge separation at semiconductor/liquid interfaces. Accounts of Chemical Research 23, 176-183 (1990).

239 Cummings, C. Y., Marken, F., Peter, L. M., Upul Wijayantha, K. G. \& Tahir, A. A. New Insights into Water Splitting at Mesoporous $\alpha$-Fe2O3 Films: A Study by Modulated Transmittance and Impedance Spectroscopies. Journal of the American Chemical Society 134, 1228-1234, doi:10.1021/ja209530s (2012).

240 Ding, Q. et al. Unravelling the water oxidation mechanism on $\mathrm{NaTaO}$ 3-based photocatalysts. Journal of Materials Chemistry A 8, 6812-6821 (2020).

241 Pham, H. H., Cheng, M.-J., Frei, H. \& Wang, L.-W. Surface Proton Hopping and Fast-Kinetics Pathway of Water Oxidation on Co3O4 (001) Surface. ACS Catalysis 6, 5610-5617, doi:10.1021/acscatal.6b00713 (2016).

242 Mesa, C. A., Rao, R. R., Francàs, L., Corby, S. \& Durrant, J. R. Reply to: Questioning the rate law in the analysis of water oxidation catalysis on haematite photoanodes. Nature Chemistry 12, 1099-1101, doi:10.1038/s41557-020-00570-5 (2020).

243 Mesa, C. A. et al. Impact of the Synthesis Route on the Water Oxidation Kinetics of Hematite Photoanodes. The Journal of Physical Chemistry Letters, 7285-7290, doi:10.1021/acs.jpclett.0c02004 (2020).

244 Zhang, Y. et al. Rate-Limiting O-O Bond Formation Pathways for Water Oxidation on Hematite Photoanode. Journal of the American Chemical Society 140, 3264-3269, doi:10.1021/jacs.7b10979 (2018).

245 Vinyard, D. J. \& Brudvig, G. W. Progress Toward a Molecular Mechanism of Water Oxidation in Photosystem II. Annual Review of Physical Chemistry 68, 101-116, doi:10.1146/annurevphyschem-052516-044820 (2017).

246 Siegbahn, P. E. M. O-O Bond Formation in the S4 State of the Oxygen-Evolving Complex in Photosystem II. Chemistry - A European Journal 12, 9217-9227, doi:10.1002/chem.200600774 (2006).

247 Siegbahn, P. E. M. Water oxidation mechanism in photosystem II, including oxidations, proton release pathways, $\mathrm{O}-\mathrm{O}$ bond formation and $\mathrm{O} 2$ release. Biochimica et Biophysica Acta (BBA) - Bioenergetics 1827, 1003-1019, doi:https://doi.org/10.1016/j.bbabio.2012.10.006 (2013).

248 Pecoraro, V. L., Baldwin, M. J., Caudle, M. T., Hsieh, W. Y. \& Law, N. A. A proposal for water oxidation in photosystem II. Pure and Applied Chemistry 70, 925-929, doi:https://doi.org/10.1351/pac199870040925 (1998).

249 Vrettos, J. S., Limburg, J. \& Brudvig, G. W. Mechanism of photosynthetic water oxidation: combining biophysical studies of photosystem II with inorganic model chemistry. Biochimica et Biophysica Acta (BBA) - Bioenergetics 1503, 229-245, doi:https://doi.org/10.1016/S00052728(00)00214-0 (2001).

250 Sproviero, E. M., Gascón, J. A., McEvoy, J. P., Brudvig, G. W. \& Batista, V. S. Quantum Mechanics/Molecular Mechanics Study of the Catalytic Cycle of Water Splitting in Photosystem II. Journal of the American Chemical Society 130, 3428-3442, doi:10.1021/ja076130q (2008).

251 Barber, J. A mechanism for water splitting and oxygen production in photosynthesis. Nature Plants 3, 17041, doi:10.1038/nplants.2017.41 (2017).

252 Surendranath, Y., Kanan, M. W. \& Nocera, D. G. Mechanistic Studies of the Oxygen Evolution Reaction by a Cobalt-Phosphate Catalyst at Neutral pH. Journal of the American Chemical Society 132, 16501-16509, doi:10.1021/ja106102b (2010).

253 Brodsky, C. N. et al. In situ characterization of cofacial $\mathrm{Co}$ (IV) centers in $\mathrm{Co}_{4} \mathrm{O}_{4}$ cubane: Modeling the high-valent active site in oxygen-evolving catalysts. Proceedings of the National Academy of Sciences 114, 3855, doi:10.1073/pnas.1701816114 (2017). 
254 Diaz-Morales, O., Ferrus-Suspedra, D. \& Koper, M. T. M. The importance of nickel oxyhydroxide deprotonation on its activity towards electrochemical water oxidation. Chemical Science 7, 2639-2645, doi:10.1039/C5SC04486C (2016).

255 Bediako, D. K., Surendranath, Y. \& Nocera, D. G. Mechanistic Studies of the Oxygen Evolution Reaction Mediated by a Nickel-Borate Thin Film Electrocatalyst. Journal of the American Chemical Society 135, 3662-3674, doi:10.1021/ja3126432 (2013).

256 Corby, S. et al. Separating bulk and surface processes in NiOx electrocatalysts for water oxidation. Sustainable Energy \& Fuels, doi:10.1039/DOSE00977F (2020).

257 Huynh, M., Bediako, D. K. \& Nocera, D. G. A Functionally Stable Manganese Oxide Oxygen Evolution Catalyst in Acid. Journal of the American Chemical Society 136, 6002-6010, doi:10.1021/ja413147e (2014).

258 Pavlovic, Z., Ranjan, C., van Gastel, M. \& Schlögl, R. The active site for the water oxidising anodic iridium oxide probed through in situ Raman spectroscopy. Chemical Communications 53, 12414-12417, doi:10.1039/C7CC05669A (2017).

259 Massué, C. et al. Reactive Electrophilic Ol- Species Evidenced in High-Performance Iridium Oxohydroxide Water Oxidation Electrocatalysts. ChemSusChem 10, 4786-4798, doi:10.1002/cssc.201701291 (2017).

260 Zhao, Y. et al. Stable iridium dinuclear heterogeneous catalysts supported on metal-oxide substrate for solar water oxidation. Proceedings of the National Academy of Sciences 115, 2902, doi:10.1073/pnas.1722137115 (2018).

261 Li, W. et al. Hematite-Based Solar Water Splitting in Acidic Solutions: Functionalization by Mono- and Multilayers of Iridium Oxygen-Evolution Catalysts. Angewandte Chemie International Edition 54, 11428-11432, doi:10.1002/anie.201504427 (2015).

262 Diaz-Morales, O., Hersbach, T. J. P., Hetterscheid, D. G. H., Reek, J. N. H. \& Koper, M. T. M. Electrochemical and Spectroelectrochemical Characterization of an Iridium-Based Molecular Catalyst for Water Splitting: Turnover Frequencies, Stability, and Electrolyte Effects. Journal of the American Chemical Society 136, 10432-10439, doi:10.1021/ja504460w (2014).

263 Shinagawa, T., Garcia-Esparza, A. T. \& Takanabe, K. Insight on Tafel slopes from a microkinetic analysis of aqueous electrocatalysis for energy conversion. Scientific Reports $\mathbf{5}$, 13801, doi:10.1038/srep13801 (2015).

264 Ooka, H., Takashima, T., Yamaguchi, A., Hayashi, T. \& Nakamura, R. Element strategy of oxygen evolution electrocatalysis based on in situ spectroelectrochemistry. Chemical Communications 53, 7149-7161, doi:10.1039/C7CC02204B (2017).

265 Ooka, H., Yamaguchi, A., Takashima, T., Hashimoto, K. \& Nakamura, R. Efficiency of Oxygen Evolution on Iridium Oxide Determined from the $\mathrm{pH}$ Dependence of Charge Accumulation. The Journal of Physical Chemistry C 121, 17873-17881, doi:10.1021/acs.jpcc.7b03749 (2017).

266 Nong, H. N. et al. Key role of chemistry versus bias in electrocatalytic oxygen evolution. Nature 587, 408-413, doi:10.1038/s41586-020-2908-2 (2020).

267 Rao, R. R., Stephens, I. E. L. \& Durrant, J. R. Understanding What Controls the Rate of Electrochemical Oxygen Evolution. Joule in press, doi:https://doi.org/10.1016/j.joule.2020.12.017 (2020).

268 Mesa, C. A. et al. Kinetics of Photoelectrochemical Oxidation of Methanol on Hematite Photoanodes. Journal of the American Chemical Society 139, 11537-11543, doi:10.1021/jacs.7b05184 (2017).

269 Natarajan, A., Oskam, G. \& Searson, P. C. The Potential Distribution at the Semiconductor/Solution Interface. The Journal of Physical Chemistry B 102, 7793-7799, doi:10.1021/jp980921k (1998).

270 Peter, L. M. Energetics and kinetics of light-driven oxygen evolution at semiconductor electrodes: the example of hematite. Journal of Solid State Electrochemistry 17, 315-326, doi:10.1007/s10008-012-1957-3 (2013). 
271 Uosaki, K. Effects of the Helmholtz Layer Capacitance on the Potential Distribution at Semiconductor/Electrolyte Interface and the Linearity of the Mott-Schottky Plot. Journal of The Electrochemical Society 130, 895, doi:10.1149/1.2119853 (1983).

272 Zandi, O. \& Hamann, T. W. Determination of photoelectrochemical water oxidation intermediates on haematite electrode surfaces using operando infrared spectroscopy. Nature chemistry 8, 778-783 (2016).

273 Boettcher, S. W. \& Surendranath, Y. Heterogeneous electrocatalysis goes chemical. Nature Catalysis, doi:10.1038/s41929-020-00570-1 (2021).

274 Kern, J. et al. Taking snapshots of photosynthetic water oxidation using femtosecond X-ray diffraction and spectroscopy. Nature Communications 5, 4371, doi:10.1038/ncomms5371 (2014).

275 Zhang, M., de Respinis, M. \& Frei, H. Time-resolved observations of water oxidation intermediates on a cobalt oxide nanoparticle catalyst. Nature Chemistry 6, 362-367, doi:10.1038/nchem.1874 (2014).

276 Sivasankar, N., Weare, W. W. \& Frei, H. Direct Observation of a Hydroperoxide Surface Intermediate upon Visible Light-Driven Water Oxidation at an Ir Oxide Nanocluster Catalyst by Rapid-Scan FT-IR Spectroscopy. Journal of the American Chemical Society 133, 1297612979, doi:10.1021/ja205300a (2011).

277 Frei, H. Water oxidation investigated by rapid-scan FT-IR spectroscopy. Current Opinion in Chemical Engineering 12, 91-97, doi:https://doi.org/10.1016/i.coche.2016.04.001 (2016).

278 Dickens, C. F., Kirk, C. \& Nørskov, J. K. Insights into the Electrochemical Oxygen Evolution Reaction with ab Initio Calculations and Microkinetic Modeling: Beyond the Limiting Potential Volcano. The Journal of Physical Chemistry C 123, 18960-18977, doi:10.1021/acs.jpcc.9b03830 (2019).

279 Plaisance, C. P., Beinlich, S. D. \& Reuter, K. Kinetics-Based Computational Catalyst Design Strategy for the Oxygen Evolution Reaction on Transition-Metal Oxide Surfaces. The Journal of Physical Chemistry C 123, 8287-8303, doi:10.1021/acs.jpcc.8b08549 (2019).

280 Mefford, J. T., Zhao, Z., Bajdich, M. \& Chueh, W. C. Interpreting Tafel behavior of consecutive electrochemical reactions through combined thermodynamic and steady state microkinetic approaches. Energy \& Environmental Science 13, 622-634, doi:10.1039/C9EE02697E (2020).

281 Klahr, B., Gimenez, S., Zandi, O., Fabregat-Santiago, F. \& Hamann, T. Competitive Photoelectrochemical Methanol and Water Oxidation with Hematite Electrodes. ACS Applied Materials \& Interfaces 7, 7653-7660, doi:10.1021/acsami.5b00440 (2015).

282 Wahl, A. et al. Photoelectrochemical studies pertaining to the activity of TiO2 towards photodegradation of organic compounds. Journal of Electroanalytical Chemistry 396, 41-51, doi:https://doi.org/10.1016/0022-0728(95)04023-H (1995).

283 Pendlebury, S. R. et al. Dynamics of photogenerated holes in nanocrystalline alpha-Fe2O3 electrodes for water oxidation probed by transient absorption spectroscopy. Chem Commun (Camb) 47, 716-718, doi:10.1039/c0cc03627g (2011).

284 Ahmed, A. Y., Kandiel, T. A., Ivanova, I. \& Bahnemann, D. Photocatalytic and photoelectrochemical oxidation mechanisms of methanol on TiO2 in aqueous solution. Applied Surface Science 319, 44-49, doi:https://doi.org/10.1016/i.apsusc.2014.07.134 (2014).

285 Shi, X. et al. Understanding activity trends in electrochemical water oxidation to form hydrogen peroxide. Nature Communications 8, 701, doi:10.1038/s41467-017-00585-6 (2017).

286 Siahrostami, S., Li, G.-L., Viswanathan, V. \& Nørskov, J. K. One- or Two-Electron Water Oxidation, Hydroxyl Radical, or H2O2 Evolution. The Journal of Physical Chemistry Letters 8, 1157-1160, doi:10.1021/acs.jpclett.6b02924 (2017).

287 Cha, H. G. \& Choi, K.-S. Combined biomass valorization and hydrogen production in a photoelectrochemical cell. Nature Chemistry 7, 328-333, doi:10.1038/nchem.2194 (2015). 
$288 \mathrm{Li}, \mathrm{T}$. et al. Photoelectrochemical oxidation of organic substrates in organic media. Nature Communications 8, 390, doi:10.1038/s41467-017-00420-y (2017).

289 You, B., Liu, X., Jiang, N. \& Sun, Y. A General Strategy for Decoupled Hydrogen Production from Water Splitting by Integrating Oxidative Biomass Valorization. Journal of the American Chemical Society 138, 13639-13646, doi:10.1021/jacs.6b07127 (2016).

290 Uekert, T., Kuehnel, M. F., Wakerley, D. W. \& Reisner, E. Plastic waste as a feedstock for solar-driven H 2 generation. Energy \& Environmental Science 11, 2853-2857 (2018).

291 Wakerley, D. W. et al. Solar-driven reforming of lignocellulose to $\mathrm{H} 2$ with a $\mathrm{CdS} / \mathrm{CdO} \mathrm{x}$ photocatalyst. Nature Energy 2, 17021 (2017).

292 Du, J., Chen, Z., Chen, C. \& Meyer, T. J. A Half-Reaction Alternative to Water Oxidation: Chloride Oxidation to Chlorine Catalyzed by Silver Ion. Journal of the American Chemical Society 137, 3193-3196, doi:10.1021/jacs.5b00037 (2015).

293 Zhang, L. et al. Photoelectrocatalytic arene C-H amination. Nature catalysis 2, 366-373 (2019).

294 Jiang, C., Moniz, S. J. A., Wang, A., Zhang, T. \& Tang, J. Photoelectrochemical devices for solar water splitting - materials and challenges. Chemical Society Reviews, doi:10.1039/C6CS00306K (2017).

295 Liao, P. \& Carter, E. A. New concepts and modeling strategies to design and evaluate photoelectro-catalysts based on transition metal oxides. Chemical Society Reviews 42, 2401-2422 (2013).

296 Pham, T. A., Ping, Y. \& Galli, G. Modelling heterogeneous interfaces for solar water splitting. Nat Mater 16, 401-408, doi:10.1038/nmat4803 (2017).

297 Katayama, K. Photo-excited charge carrier imaging by time-resolved pattern illumination phase microscopy. The Journal of Chemical Physics 153, 054201, doi:10.1063/5.0009312 (2020). 